\title{
Model-based analysis of continuous crystallization/reaction processes separating conglomerate forming enantiomers
} DOI:

10.1021/acs.cgd.6b01487

\section{Document Version}

Accepted author manuscript

Link to publication record in Manchester Research Explorer

Citation for published version (APA):

Köllges, T., \& Vetter, T. (2016). Model-based analysis of continuous crystallization/reaction processes separating conglomerate forming enantiomers. Crystal Growth and Design. https://doi.org/10.1021/acs.cgd.6b01487

\section{Published in:}

Crystal Growth and Design

\section{Citing this paper}

Please note that where the full-text provided on Manchester Research Explorer is the Author Accepted Manuscript or Proof version this may differ from the final Published version. If citing, it is advised that you check and use the publisher's definitive version.

\section{General rights}

Copyright and moral rights for the publications made accessible in the Research Explorer are retained by the authors and/or other copyright owners and it is a condition of accessing publications that users recognise and abide by the legal requirements associated with these rights.

\section{Takedown policy}

If you believe that this document breaches copyright please refer to the University of Manchester's Takedown Procedures [http://man.ac.uk/04Y6Bo] or contact uml.scholarlycommunications@manchester.ac.uk providing relevant details, so we can investigate your claim.

\section{OPEN ACCESS}




\title{
Model-based analysis of continuous
}

\section{crystallization/reaction processes separating conglomerate forming enantiomers}

\author{
Till Köllges and Thomas Vetter* \\ School of Chemical Engineering and Analytical Science, The University of Manchester, \\ Manchester, UK \\ E-mail: thomas.vetter@manchester.ac.uk \\ Phone: +44 1613064370
}

\begin{abstract}
The separation conglomerate forming chiral molecules is investigated in continuous processes combining crystallization, milling and a racemization reaction in solution. Two process configurations that increase enantiomeric purity starting from racemic mixtures are presented and assessed with respect to attainable enantiopurities, achievable yields and productivities. The two processes differ in their feeding strategy: one is fed with a supersaturated solution of racemic composition and one is fed with a racemic mixture of solids. The two cases are studied using a consistent mathematical model based on population balance equations. A parameteric analysis on the key operating parameters of the processes is conducted and operating regions leading to high enantiomeric purity are identified. By combining the crystallization $/ \mathrm{mill} /$ reaction process with appropriate filtration and solvent removal units, as well as recycle operations, we show that full enantiomeric purity at $100 \%$ yield is theoretically attainable for the solution-fed process, while it is unattainable for the solid-fed process variant.
\end{abstract}




\section{Introduction}

Enantiomeric purity is paramount for many products in the food, agrochemical and pharmaceutical industry, because the two (or more) different enantiomers can have different effects on the environment or the human body. These different effects of enantiomers result from interactions of chiral molecules with chiral receptors that are frequently found in living organisms. One of the most widely known tragedies in this context concerns thalidomide, which was heavily prescribed in the 1960's as a medication inducing deep sleep and preventing morning sickness in pregnant women (these positive effects are attributable to the R-enantiomer). Tragically, the racemic mixture of thalidomide led to the stillbirth and malformation of children when taken during pregnancy.* Increasing regulatory action regarding racemic active ingredients has led the pharmaceutical industry to strive towards enantiomerically pure products with an increasing number of new molecular entities produced as pure enantiomers. ${ }^{3}$

Enantiopurity can be reached directly by asymmetric synthesis or by an additional purification step, e.g., column chromatography ${ }^{4}$, diastereomeric salt formation ${ }^{5,6}$, co-crystallization with chiral coformers ${ }^{7}$ or by using chiral membranes. ${ }^{8}$ A review of techniques is provided elsewhere. ${ }^{9}$ While asymmetric synthesis is attractive, since the formation of undesired enantiomers is avoided, achieving very high enantiomeric purities is not always possible or requires the use of (often themselves toxic) metal catalysts. Separation methods are therefore prevalent when high enantiomeric excesses are desired in the final product. The selection of a suitable resolution process depends strongly on the physical properties of the compound. For instance, it is not always possible to form a diastereomeric salt. Furthermore, some separation processes, e.g., column chromatography or membrane processes result in a liquid product and at least one further unit operation is necessary to obtain a typically desirable solid product. Crystallization processes that directly lead to an enantiomerically pure prod-

${ }^{*}$ Note that thalidomide enantiomers are known to rapidly racemize in vivo ${ }^{1}$, so that administering only the desired enantiomer would still likely have led to teratogenic effects. Nonetheless, thalidomide has seen a renaissance as a drug to manage certain types of cancer in recent years. ${ }^{2}$ 
uct in solid form are therefore an attractive option for cost, but also quality reasons. Whether and how to run such a process is chiefly governed by the underlying thermodynamics, i.e., the ternary phase diagram of the two enantiomers and the solvent. The three most commonly encountered ternary phase diagrams of chiral molecules are introduced in Figure 1.
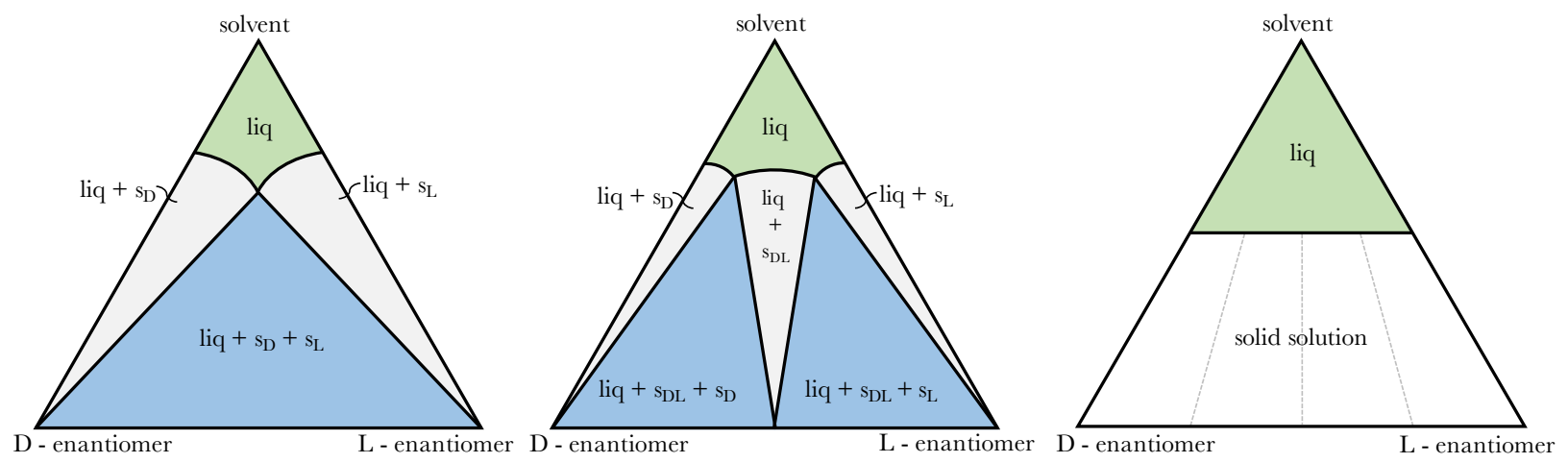

Figure 1: Ternary phase diagrams of a conglomerate (left), a racemate (middle) and a solid solution (right). The one phase regions are shown in green, the two phase regions in grey and the three phase regions in blue.

The systems of chiral molecules and solvent shown in that figure crystallize (from left to right) either as conglomerates, as racemic compounds or as solid solutions. The term conglomerate describes the behavior that an enatiomer crystallizes only with molecules of the same handedness in a crystal lattice, e.g., $s_{\mathrm{D}}$ and $s_{\mathrm{L}}$. While it is possible to have both $\mathrm{D}$ and L crystals and a liquid phase present at the same time (blue region), each individual crystal is enantiopure. A mixed crystal lattice, e.g. $s_{\mathrm{DL}}$, does not form, contrary to the case of racemic compound forming systems, where a 1:1 mixture of enantiomers exists in a stable crystal structure, and solid solutions, where the ratio of enantiomers in the solid varies. Conglomerate forming systems, which are estimated to make up roughly $10 \%$ of chiral systems,${ }^{6}$ are of special interest, because they allow to conveniently obtain enantiopure solids even when the liquid phase is a racemic mixture. The process of obtaining enantiopure solids from a racemic starting point is typically referred to as deracemization.

It has been shown that conglomerates can be separated via preferential crystallization where the process is operated in the three phase region. ${ }^{10,11}$ Briefly described, preferential 
crystallization works by adding crystals of one enantiomer to a supersaturated solution, which then preferentially grow by incorporating molecules of the same chirality from the solution. Since this process is carried out in the three phase region, its thermodynamic equilibrium (the top apex of the blue phase region) exhibits a racemic composition in solution. If one started the process from a racemic solution composition at higher solute concentration (vertical line through the apex), the solids in that thermodynamic equilibrium are a racemic mixture as well (apart from the originally added seed crystals). However, if the process is operated in a way that avoids nucleation of crystals of the undesired enantiomer (i.e., necessarily away from the thermodynamic equilibrium), enantiopure product can be obtained. Recently, preferential crystallization processes with two coupled crystallizers have been presented that yield one enantiomer in pure form in each crystallizer. ${ }^{12,13}$ The coupled crystallizers were also operated as mixed suspension mixed product removal crystallizers (MSMPRC), fed with a supersaturated solution, representing a continuous process configuration. ${ }^{14}$ Such processes need to be be controlled in (at least) two ways: first, the nucleation of the respectively undesired enantiomer must be avoided, e.g., by reducing the supersaturation of the undesired enantiomer in each crystallizer. This can, for example, be accomplished by exchanging the liquid phases of the coupled crystallizers. ${ }^{14-17}$ An alternative is to employ a racemization reaction to avoid an excess of the undesired enantiomer in the liquid phase. ${ }^{18}$ If this reaction is sufficiently fast, the liquid phase will essentially remain at racemic composition, even though one enantiomer is preferentially crystallized. Clearly, the latter strategy has the benefit that it allows converting the undesired enantiomer to the desired one in solution; a process for which crystallization constantly provides a driving force. Essentially, while separation processes can only reach 50\% yield (perfect separation of two enantiomers from racemic composition), employing the reaction allows a theoretical yield of $100 \%$ (perfect separation of enantiomers and conversion of the undesired enantiomer to the desired one). Second, for continuous processes one must maintain a particle size distribution in the crystallizer that provides enough surface area to enable substantial crystal growth and therefore achieve a 
high yield. While it is possible to rely on secondary nucleation to generate new crystals of the same chirality (e.g., through attrition caused by crystal/crystal collisions and crystal/stirrer collisions), more controllable and efficient approaches have ben realized to achieve this. For instance continuous seeding ${ }^{14-16}$, generating small crystals via suspension mills ${ }^{17}$ or the use of ultrasound to shatter crystals into smaller fragments ${ }^{19}$ have all been described in the literature.

Kondepudi et al. ${ }^{20}$ reported that for the case of sodium chlorate enantiopurity in the crystalline phase can also be achieved in batch slurry experiments operated in the absence of significant supersaturation levels. ${ }^{\dagger}$ Viedma $^{21}$ reported that stirred slurry experiments performed in the presence of glass beads, starting from a slight enantiomeric enrichment in the solid phase, reach full enantiomeric purity after some time. Furthermore, it was shown that the process can be driven towards either enantiomer by choosing the enantiomer initially in excess in the solid phase. After further experimental evidence was gathered on achiral molecules, such as $\mathrm{NaBrO}_{3}{ }^{22}$ and ethylenediammonium sulfate ${ }^{23}$, Noorduin et al. ${ }^{24}$ tested the approach on the chiral imine of 2-methyl-benzaldehyde and phenylglycinamide. In contrast to the achiral molecules used by Kondepudi et al. ${ }^{20}$ and Viedma ${ }^{21}$, the molecule employed by Noorduin et al. ${ }^{24}$ was itself chiral, meaning that it keeps its chirality in the dissolved state. However, the authors introduced a racemizing agent (an organic base in this case) allowing the interconversion of enantiomers. Considerable effort was devoted to elucidate the underlying mechanisms of the overall process ${ }^{25-28}$, which was identified to consist of a complex interplay of the racemization reaction, crystal attrition, Ostwald ripening and agglomeration. In light of Viedma's significant contributions towards a mechanistic understanding of this process, Noorduin et al. ${ }^{25}$ suggested to name it "Viedma ripening".

From the above discussion of preferential crystallization and Viedma ripening, it is noteworthy that the two processes exhibit considerable similarities. In fact, the underlying thermodynamics and kinetics must remain the same for a given combination of enantiomers,

\footnotetext{
${ }^{\dagger}$ Note that sodium chlorate is achiral in solution, but exhibits chirality in the solid state and crystallizes as a conglomerate.
} 
solvent, and racemizing agent (at the same concentration). ${ }^{\ddagger}$ However, the two processes differ in the fact whether solids of the undesired enantiomer are present (Viedma ripening) or absent (preferential crystallization). While a few studies on continuous preferential crystallization have been reported, ${ }^{14-17}$ investigations on continuous Viedma ripening processes have not yet been reported to the best of our knowledge. Among the perceived advantages of continuous processes are i) their operation at a (stable) steady state, making them easier to control and thus more robust, ii) potentially smaller reactor scales at the same productivity as equivalent batch processes, ${ }^{17,29}$ iii) the absence of batch to batch product variations ${ }^{30}$, as well as iv) lower manual handling requirements overall. The operation at a stable steady state carries considerable merit for preferential crystallization specifically, as such processes -in contrast to batch preferential crystallization processes - are able to recover from process disturbances quite readily, as shown for example by Vetter et al. ${ }^{17}$.

In this work, we evaluate continuous Viedma ripening processes and preferential crystallization processes that additionally involve a racemization reaction in terms of their ability to reach an enantiopure solid product, as well as their operational characteristics and limitations. Furthermore, when designing the continuous processes presented in this paper, we strive to achieve high yields and minimize product and solvent losses. To this end, we do not limit ourselves only to crystallizer units, but will couple them to additional unit operations that enable recycle operations, as well as filtration units for liquid/solid separation. These flowsheets and their rationale are presented in the following section. Since there is a good mechanistic understanding of Viedma ripening and preferential crystallization processes available in the literature, the process performance can be evaluated through the use of a detailed, mechanistic process model. Our emphasis in using this model is to compare the two process variants on an equal footing, i.e., with the same kinetics and the same underlying assumptions. To keep the intensity of secondary nucleation high and equal, a suspension

\footnotetext{
$\ddagger$ The introduction of a racemizing agent into the liquid phase makes the mixture a quaternary system. However, at conditions where no solids including the racemizing agent form, the system can be treated as pseudo-ternary and when the racemizing agent is achiral (as in all cases reported so far in the literature) the phase diagram in Figure 1 remains symmetric.
} 
mill is implemented in both processes. The model is introduced in the section following the presentation of flowsheets. The model is then used to investigate the process start-up and stable steady states are revealed. By conducting an extensive parameter study, we uncover how the process reacts to changes in operating conditions and highlight regions of operating parameters that lead to high enantiomeric purities. The applicability of the study to other related cases is discussed in the penultimate section before ending with concluding remarks.

\section{Continuous Process Flowsheets}

In order to realize a continuous preferential crystallization and Viedma ripening process, we present two different process layouts as shown in Figure 2. In both configurations, a single MSMPRC is used for the purpose of crystallization and the concomittant racemization reaction. The MSMPRC is continuously fed with supersaturated racemic solution (flowsheet A) or a racemic composition of solids (flowsheet B).

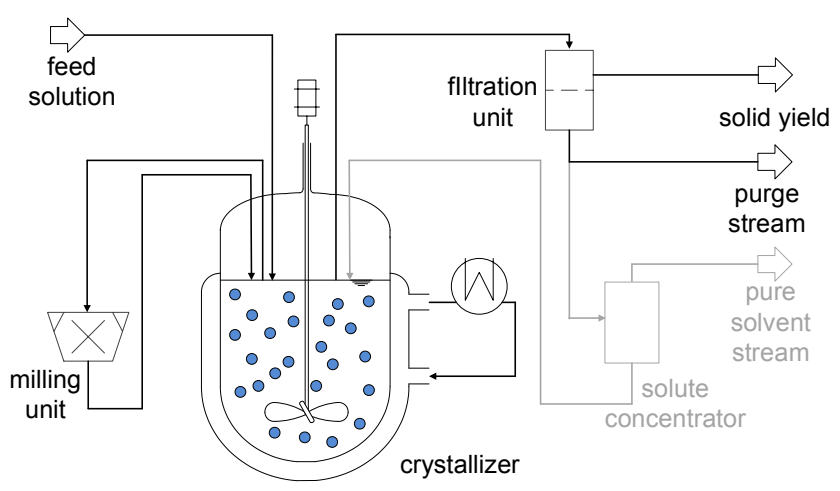

(A)

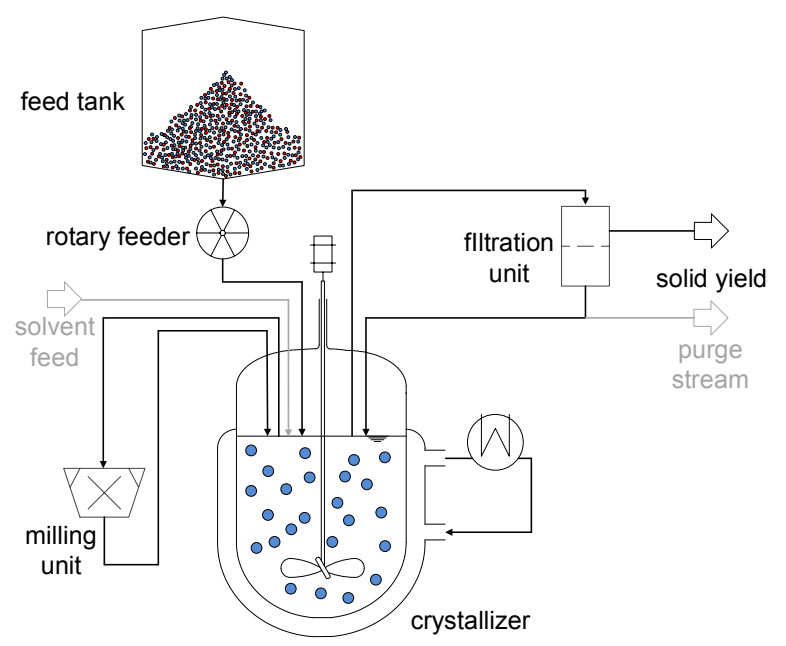

(B)

Figure 2: (A) Solution-fed process flowsheet (preferential crystallization) (B) solid-fed flowsheet (Viedma ripening). The grayed out parts in flowsheet (A) are optional improvements to accomplish increased yields. In both flowsheets purge streams may be included to avoid the build up of impurities (if necessary).

Focusing first on flowsheet A, the crystallizer is started up with an excess of crystals 
of the desired enantiomer present. Several studies ${ }^{14-17}$ have shown that a proper choice of operating conditions can lead to a steady state delivering an enantiopure product under this feeding strategy. In such a steady state, the liquid phase remains slightly supersaturated. The extent of supersaturation at steady state is influenced by the feed concentration, the residence time, as well as the surface area of the crystals present in the MSMPRC. For a given feed concentration, longer residence times and higher overall surface areas of the crystals lead to a lower supersaturation at steady state. For flowsheet A to be more effective, new crystals of the desired enantiomer need to be introduced or formed in the process continuously, because part of the crystals are leaving the crystallizer continuously as well. Several ways to accomplish this were presented in the literature, among them continuously seeding the process with crystals of the desired enantiomer Qamar et al. ${ }^{15}$, which then grow in the crystallizer and are subsequently removed. Such an operating strategy would represent a hybrid between flowsheet A and B in Figure 2, whereas in flowsheet B a racemic mixture is fed. Alternatively, as presented in Vetter et al. ${ }^{17}$ and depicted in Figure 2, a suspension mill connected in a loop to the MSMPRC can be used. In this way, the generation of new crystals is tunable to a desired level by adjusting operating parameters of the mill, as well as the flow rate through it. ${ }^{31}$ Combined, we will refer to this as the "milling intensity" in the following. $\S$ When starting with an excess of crystals of the desired enantiomer in the crystallizer, the suspension mill will generate a higher number of crystals of the desired enantiomer in comparison to the undesired enantiomer, which can ultimately drive the process towards enantiopurity. ${ }^{17}$ However, the preferential crystallization of the desired enantiomer leads to the liquid phase being relatively enriched in the undesired enantiomer, hence nucleation would be more likely for the undesired enantiomer than for the desired enantiomer. Avoiding nucleation in a preferential crystallization process is an important consideration, because primary nucleation of the undesired enantiomer would impact the product purity negatively. For the cases discussed in this work, the build up of supersaturation of the

\footnotetext{
$\S$ Note that the milling intensity is of course not infinitely tunable, but is constrained by physical limits, e.g., the minimal particle size obtainable in a certain type of mill and limits on the throughput of the mill.
} 
undesired enantiomer (and hence nucleation) is avoided by having a racemization catalyst present in solution that removes some or all of the enantiomeric excess from the liquid phase (depending on the reaction kinetics). Secondary nucleation on the other hand would benefit a continuous preferential crystallization process, because it would favor the crystals of the enantiomer already present in excess, i.e., of the desired one.

From the process description given above, we can conceptually analyze how the overall process reacts to changes in operational characteristics and even the underlying kinetics. Specifically, we can surmise that the composition of the liquid phase at steady state must fall within a triangular region of the ternary phase diagram (for any kinetics and operational characteristics), drawn as the purple region in Figure 3, where we have assumed that the L-enantiomer is the desired one. This region is defined by a thermodynamic boundary (solid line), a mass balance line and the line at racemic composition (the latter two are shown as dashed lines). The composition of the feed stream is indicated as a square and an exemplary steady state is shown as a circle. Steady states with a racemic solid phase always occur in the presence of racemic liquid phases (i.e., they are located on the vertical dashed line), while steady states with an enantiomerically enriched solid phase can be located anywhere in the purple region (including on the vertical dashed line). Steady states with an enantiopure solid phase in the absence of a racemization reaction would be located on the dashed mass balance line. The exemplary steady state drawn in Figure 3 is therefore consistent with a steady state that is at least enantiomerically enriched in the solid phase. For the sake of argument, we assume that the steady state is enantiomerically pure in the following.

Zooming in around the steady state (Figure 3 on the right), we now report how the steady state changes qualitatively when process conditions, crystal growth kinetics and racemization kinetics are changed from this reference case. An increase in crystallizer residence time, a faster crystal growth rate or an increased milling intensity leads to a lower steady state supersaturation and hence higher yield (arrow (1)). In the case of an enantiopure solid phase the arrow follows the mass balance line connecting the reference steady state and the vertex 
representing the L-enantiomer. When the crystallizer residence time is decreased, the crystal growth rate is slower or when the milling intensity is decreased, the supersaturation in the liquid phase increases and the yield decreases (arrow (2)). Faster racemization increases the amount of L-enantiomer available, which leads to a larger mass of L-enantiomer crystals with higher surface area and lower supersaturation/higher yield (arrow (3)). Slower deracemization has the opposite effect, resulting in arrow (4). Note that arrows (3) and (4) were intentionally drawn with different slopes, because the effect of a mass increase/decrease of L-crystals and the corresponding changes in surface area and supersaturation have a nonlinear effect on the system. Their precise orientation however depends on the specific kinetics. The orientations of arrow (1) and 2 on the other hand are fixed.

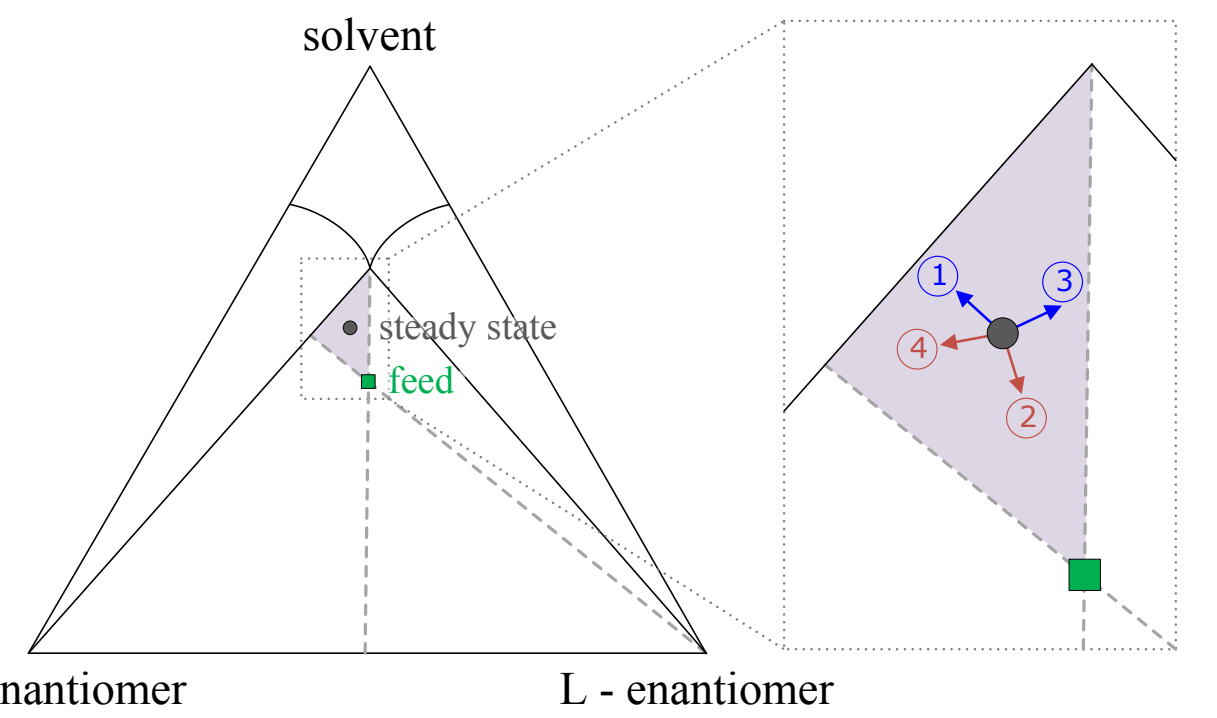

Figure 3: Ternary phase diagram with region containing all potential steady states of flowsheets A and B (cf. Figure 2) highlighted in purple. An exemplary feed point composition is drawn as a rectangle, while a reference steady state is highlighted with a circle. The zoom in displayed on the right elucidates how the reference steady state moves within the operating space when kinetics or operating parameters are changed: (1) increased residence time, faster crystal growth and/or increased milling intensity, 2 decreased residence time, slower crystal growth, and/or decreased milling intensity, 3 faster racemization reaction, (4) slower racemization reaction.

From the MSMPRC, the resulting suspension is transported to a continuous filtration unit (see, e.g., Acevedo et al. ${ }^{32}$ for an experimental implementation of such a unit) where the 
crystals and the liquid phase are separated from each other. When the process conditions (residence time, milling intensity, feed concentration, etc.) are chosen correctly, enantiopure solids are obtained at this point. The liquid phase contains remaining solute molecules (both enantiomers), solvent and the racemization catalyst. Therefore, treating it as waste would constitute a loss of product and catalyst; both are, however, valuable. In this work, we recycle the liquid stream leaving the filtration unit back into the crystallizer. However, this can only occur after some of the solvent (which entered the flowsheet through the feed stream) is removed in an ancillary processing unit (labelled "solute concentrator" in the flowsheet) as the solvent would otherwise build up within the process. Such a solvent removal unit could, for example, be a distillation column or a membrane unit. The choice between different ancillary units would be given by the physical properties of the enantiomers, solvent and racemization catalyst $\mathbb{I}$. Additionally, a purge stream is necessary if there are any impurities present in the feed stream (e.g., unconverted reactands from preceding chemical reactions) or if impurities are formed within the MSMPRC. While the use of the grayed out part in flowsheet A, i.e., the solvent concentrator and recycle stream, is optional, the use of such a unit has the potential to increase the overall process yield up to the theoretical maximum of $100 \%$. The change of steady state introduced by the recycle operation could be rationalized as before with all possible steady states lying within the purple region in Figure 3.

The continuous Viedma ripening flowsheet (B in Figure 2) employs similar principles to flowsheet $\mathrm{A}$, but is fed directly with a racemic mixture of crystals instead of a supersaturated, racemic solution. Viedma ripening is the result of an interplay of several phenomena (attrition, agglomeration, Ostwald ripening etc.) and occurs in a liquid phase that is minimally supersaturated. The mechanistic details are discussed together with a process model for it in the next section. Here, it suffices to rationalize Viedma ripening to result in the conversion of the racemic feed into an enriched outlet stream and to know that Viedma

\footnotetext{
IFor distillation enantiomers and racemization catalyst must be thermally stable and their volatility must be different from the solvent's. For a membrane separator a selective and stable membrane material must be identified. Further points are discussed in the penultimate section of this article.
} 
ripening is enhanced by abrasive grinding. The latter property makes the inclusion of a suspension mill in flowsheet B again a sensible choice. The outlet of the crystallizer is fed to a continuous filtration unit where a crystalline product is obtained. The liquid phase containing enantiomers, solvent and racemization catalyst is recycled. However, in this case no additional solvent was fed to the crystallizer, so that - if the filtration unit achieves a perfect separation of liquid and solids - all of the liquid stream can be recycled. On the other hand if the filtration unit does not achieve complete separation of crystals and liquid, a solvent make up stream must be employed. Furthermore, if the solids entering the process contain impurities or if new impurities are formed, a purge stream is necessary to avoid the build up of impurities. Steady states associated with this mode of operation will always remain close to the thermodynamic equilibrium and could be drawn accordingly in Figure 3.

While the qualitative discussion given above and conducted in the light of Figure 3 already gives considerable insight into the behavior of the processes, a quantitative assessment of solid state enantiomeric purity resulting from a given set of kinetics and operating conditions cannot be performed without a comprehensive model that accounts for all dynamic aspects of the flowsheet. Therefore, we will employ detailed process simulations using a population balance equation (PBE) model for the solid phases and corresponding mass balances for the liquid phase, as detailed in the next section. Using this model, the two flowsheets presented above are then investigated in three different operating modes in this work:

- The solution-fed process without recycle and excluding a work up of the crystallizer outlet stream (i.e., excluding the grayed out part in Figure 2A).

- The solution-fed process including the grayed recycle shown in Figure 2A, to increase the yield of the overall product stream.

- The solid-fed process in Figure 2B, where a solute concentrator is not necessary.

Parameter studies are conducted to investigate possible feed loads, residence times as well as to find operating spaces where an enantiopure product can be obtained and to determine 
the start up behavior and stability of the resulting steady states.

\section{Process model}

In order to carry out quantitative simulations of the process flowsheets presented above, a complete model must fulfill the following three critera: first, the key mechanisms involved in the crystallization process must be understood and their kinetics must be expressed by appropriate constitutive equations. Second, a modelling framework must be employed that allows tracking the evolution of the whole particle size distributions (for both enantiomers) in the crystallizer and the mill. Third, mass balances relating the solid phases and the liquid phase in the crystallizer to each other must be defined, as well as mass balances connecting the ancillary units to the crystallizer. In the following, we will address all three aspects.

\section{Mechanistic aspects and constitutive equations}

As highlighted in the introduction a considerable set of works reported over the last decade has aimed at identifying the mechanisms involved in Viedma ripening. The first models for Viedma ripening were set up by Uwaha ${ }^{33}$ and Saito and Hyuga ${ }^{34}$, describing five species: D and L crystals, D and L microclusters and achiral molecules in solution. These species were coupled with each other through rate equations including the incorporation (agglomeration) of microclusters into larger crystals of the same handedness. A further model approach by Saito and Hyuga ${ }^{35}$ suggested an asymmetric racemization reaction at the crystals' surfaces. The investigation by Noorduin et al. ${ }^{36}$ combined a similar model approach with experimental data and highlighted the fundamental mechanisms leading to solid phase deracemization: solution phase racemization, crystal agglomeration, attrition and Ostwald ripening (growth of large crystals, dissolution of small crystals). Figure 4 summarizes the interaction of these mechanisms. The crystals of the two enantiomers are drawn as separate populations to illustrate that all mechanisms occur to both enantiomers concomittantly. The solid particles 
change their size due to attrition and agglomeration and due to growth and dissolution. While attrition and agglomeration do not affect the liquid phase composition in the first approximation, the growth and dissolution mechanisms do.

Rather than (arbitrarily) assuming a fixed number of crystal sizes to be involved in the process, the PBE model by Iggland and Mazzotti ${ }^{37}$ described the evolution of the complete particle size distributions (PSDs) and included all mechanisms shown in Figure 4. The model was used to thoroughly investigate the influence of kinetic parameters and successfully reproduced trajectories and qualitative behaviors of experimental studies on Viedma ripening. Through these parameter studies it was clarified which mechanisms are indispensable for Viedma ripening to occur and which mechanisms merely influence the ripening rate. ${ }^{37}$ Some of their conclusions are echoed, for example, in the experimental and theoretical study by Gherase et al. ${ }^{38}$, who show that Viedma ripening also works excluding the agglomeration mechanism if starting crystal populations of different mean size are used. A comprehensive analysis of the influence of initial conditions was also reported in the literature. ${ }^{39}$ In recent studies the Viedma ripening process was accelerated by increasing the stress onto the crystals in different ways. ${ }^{40,41}$ Xiouras et al. ${ }^{42}$ compared abrasive grinding using glass beads to the application of ultrasound, both, to break crystals into smaller fragments and decrease the time required to reach enantiopurity. The experimental results were again qualitatively consistent with the PBE model. With respect to specific implementations of each mechanism in this article, we closely follow earlier works ${ }^{17,37,39}$ and detail the constitutive equations for each mechanism in the following. All parameters used within these equations are reported in Table 1.

Crystal growth, dissolution and Ostwald ripening: According to the Gibbs-Thompson equation (see, e.g., Mullin ${ }^{30}$ ) small particles exhibit a higher solubility than larger ones, which leads to the phenomenon of Ostwald ripening, i.e., in a population of small and large particles in contact with a liquid phase, the large particles will grow at the expense of the 


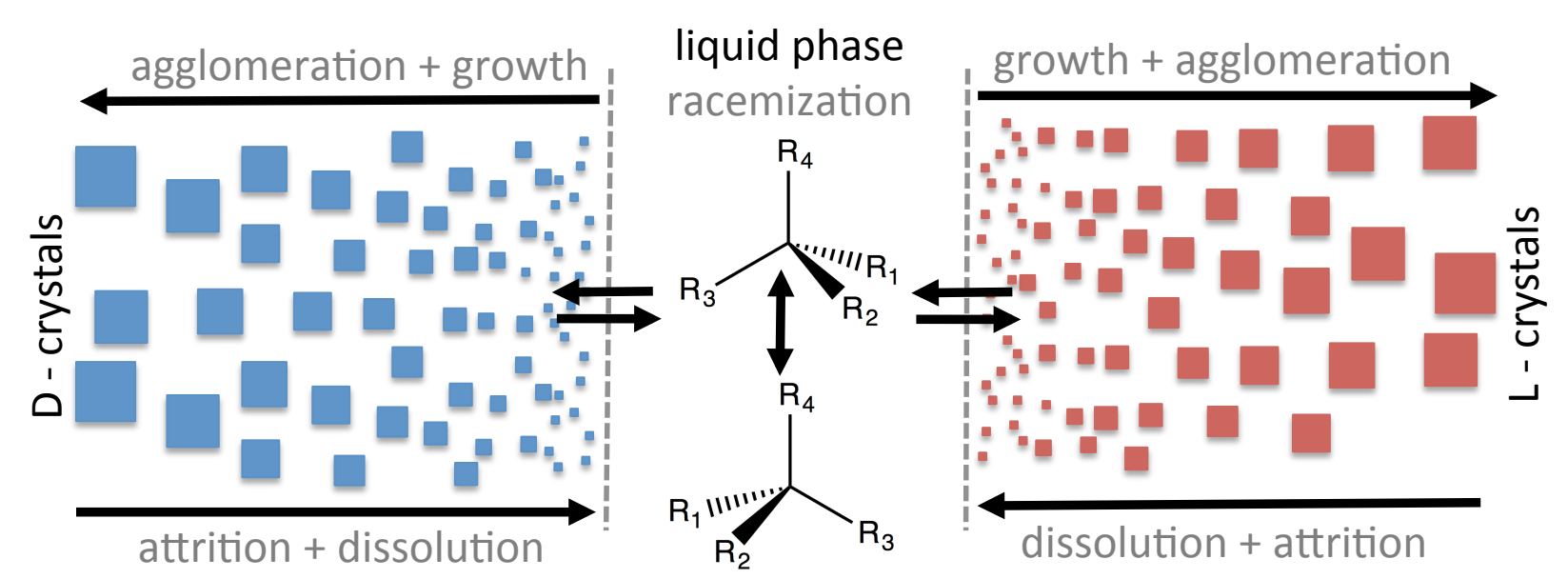

Figure 4: Key mechanisms involved in Viedma ripening and their effect on the size of the crystals involved.

small particles (which shrink and finally dissolve). ${ }^{43-45}$ Assuming that the crystals can be described using a single characteristic length, $L$, the Gibbs-Thomson equation can be derived from classical nucleation theory ${ }^{46}$ and can be formulated for the case of enantiomers as:

$$
c_{i}^{\star}\left(c_{j}, L, T\right)=c_{i, \infty}^{\star}\left(c_{j}, T\right) \exp \left(\frac{2 k_{\mathrm{a}} \gamma V_{\mathrm{m}}}{3 k_{\mathrm{v}} R T L}\right)=c_{i, \infty}^{\star}\left(c_{j}\right) \exp \left(\frac{\alpha}{L}\right) \text { with } i, j \in\{L, D\} \text { and } j \neq i
$$

where $c_{i}^{\star}\left(T, c_{j}, L\right)$ is the solubility of enantiomer $i$, which depends on the temperature, $T$, the concentration of the counter-enantiomer $c_{j}$ and the particle size $L . c_{i, \infty}^{\star}\left(c_{j}, T\right)$ is the solubility at large particle sizes, $\gamma$ is the surface energy, $k_{\mathrm{a}}$ and $k_{\mathrm{v}}$ are surface and volume shape factors, $V_{\mathrm{m}}$ is the molar volume and $R$ is the gas constant. We will investigate isothermal crystallization processes in this work, so that the temperature is constant and has been lumped into the capillary length, $\alpha$ on the right hand side of Eq. (1). Temperature dependencies will not explicitly be mentioned in subsequent equations. Note that while the explicit dependence of the solubility of one enantiomer on the other has not been accounted for in previous works $37,39,47$, we include it here, because we also consider preferential crystallization processes, which can result in steady states where the liquid phase is neither racemic, nor close to the racemic equilibrium point in the phase diagram (i.e., the apex of the three phase region in Figure 1). 
In order to describe the Ostwald ripening component within Viedma ripening, as well as the crystal growth component in preferential crystallization, we define a crystal growth rate, $G$, that depends on a crystallization driving force, so that:

$$
G\left(S_{i}, L\right)=k_{\mathrm{g}}^{\prime}\left(c_{i}-c_{i, \infty}^{\star}\left(c_{j}\right) \exp \left(\frac{\alpha}{L}\right)\right)=k_{g}\left(S_{i}-\exp \left(\frac{\alpha}{L}\right)\right)
$$

where $k_{\mathrm{g}}=k_{g}^{\prime} c_{i, \infty}^{\star}\left(c_{j}\right)$ is a kinetic parameter and the supersaturation of enantiomer $i, S_{i}$, is defined as

$$
S_{i}=\frac{c_{i}}{c_{i, \infty}^{\star}\left(c_{j}\right)}
$$

Coupled with a PBE model accounting for the evolution of PSD, such growth rate equations have been shown multiple times to accurately describe Ostwald ripening, as well as crystal growth ${ }^{37-39,45,47}$. The size-dependent solubility and the growth rate used in this work are visualized in Figure 5 for parameter values and supersaturations often encountered in preferential crystallization and Viedma ripening processes. From this figure it is noteworthy that only very small particles are subject to a negative growth rate at non-negligible supersaturations. Hence, from that figure it is already clear that Ostwald ripening will play only a minor role in preferential crystallization processes. Nonetheless, using a single set of constitutive equations puts the two process variants (solution-fed vs. solid-fed) shown in Figure 2 on equal footing and will enable an unbiased comparison.

Breakage/Attrition: A wet suspension mill was included in the process flowsheets, because particle breakage/attrition is known to speed up Viedma ripening ${ }^{21,36,38,42}$, as well as to provide a continuous means for "seed generation" in the process flowsheets ${ }^{17}$. Even in the complete absence of (primary) nucleation, the continuous breaking of particles will replace particles that are leaving the crystallizer through its outlet and hence a stable steady state can be achieved. Particle brekage can be rationalized as the product of a breakage rate (how many particles of a certain size are breaking per time) and a fragment size distribution, i.e., 
(A)

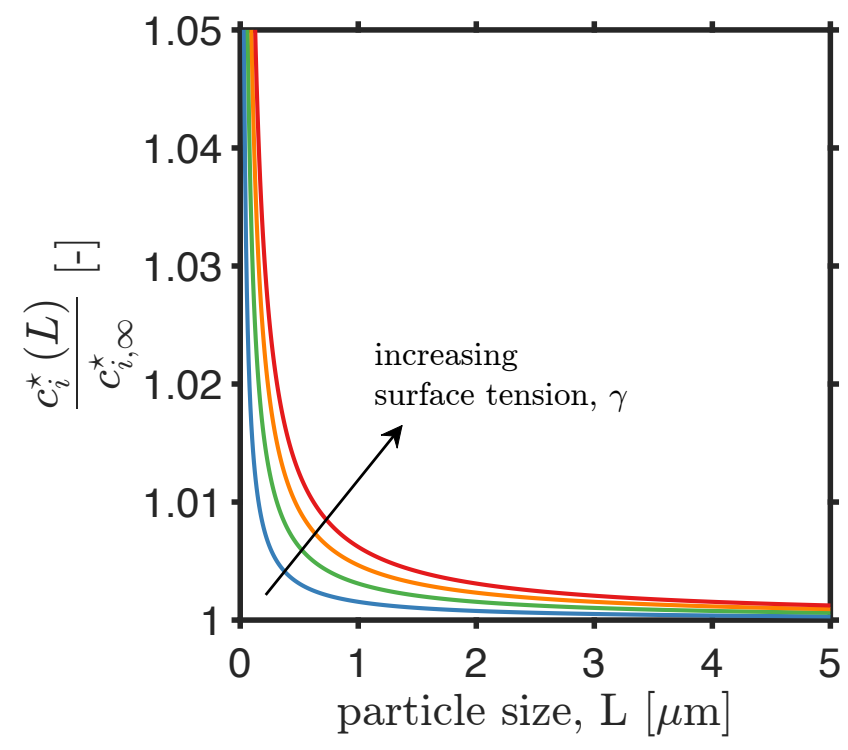

(B)

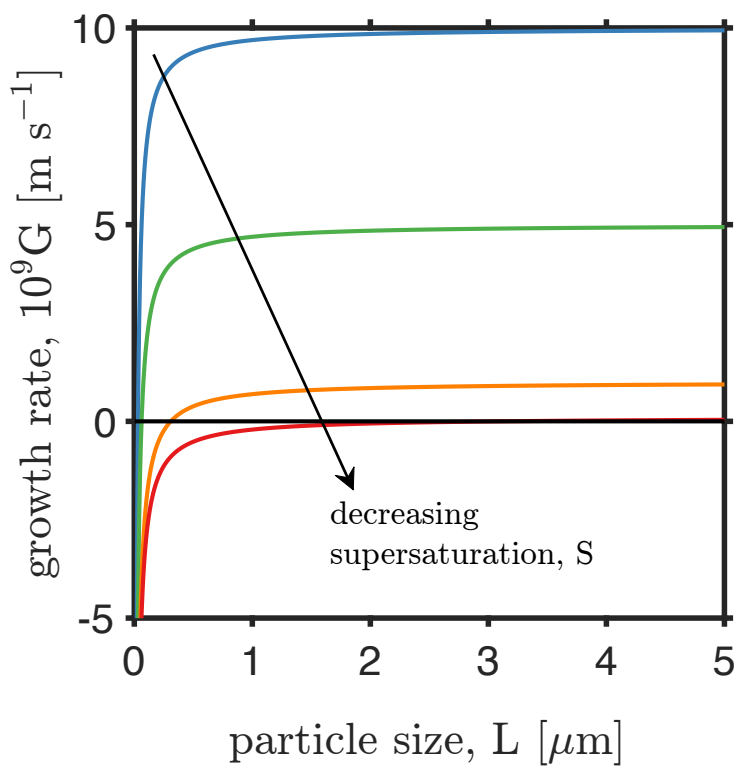

Figure 5: Visualization of size-dependent solubility and growth rate: (A) Normalized solubility vs. particle size at different surface energies. The solubility is drawn for surface energies, $\gamma$, of 1 to $4 \times 10^{-2} \mathrm{~J} \mathrm{~m}^{-2}$. (B) Growth rate $G$ vs. particle size for the same surface energies at different supersaturations $(S \in\{1.1,1.05,1.01,1.001\})$.

what size of fragments result from a particle after it underwent attrition or breakage. A variety of expressions has been developed for both contributions in the literature. Here, we choose a flexible daughter distribution $d$ taking the form ${ }^{17,37}$

$$
d(\xi, \eta)=3 \xi^{2}(2 \mathrm{q}+1)\left(\frac{2}{\eta^{2}}\right)^{2 \mathrm{q}+1}\left(\xi^{3}-\frac{\eta^{3}}{2}\right)^{2 \mathrm{q}}
$$

where $\xi$ is the size of the parent particle, $\eta$ the formed fragment and $q$ is a parameter governing the shape of the distribution. By using this description, we assumed that the fragments formed through breakage have the same shape as the parent particle. Making this assumption is common practice and results in a mathematical model that is tractable. In Eq. (4) (see also in Figure 6A) low values of $q$ result in a distribution that represents particles breaking in roughly equal-sized fragments, while high values of the parameter result in a distribution mimicking attrition (i.e., a particle breaks into a large and small fragment). The value of $q$ is dependent on the type of mill used as well as on the material properties of 
the crystals. The daughter distribution has the following mathematical properties

$$
\int_{0}^{\xi} \eta^{3} d(\xi, \eta) \mathrm{d} \eta=\xi^{3} \text { and } \int_{0}^{\xi} d(\xi, \eta) \mathrm{d} \eta=2
$$

indicating that the volume (and hence mass) of the formed fragments is the same as the volume of the original particle and that two particles are formed by a breakage event. The breakage rate, $K$, is here assumed to be dependent on particle size, but independent of suspension density:

$$
K(L)=k_{\mathrm{b}, 1} L^{k_{\mathrm{b}, 2}}
$$

where $k_{\mathrm{b}, 1}$ and $k_{\mathrm{b}, 2}$ are again specific to a material and the mill type. Note that breakage rates independent of suspension density assume that breakage is triggered through particles colliding with parts of the mill (or crystallizer). At high suspension densities, particle-particle collisions become more frequent; hence, the breakage rate would become dependent on the suspension density.

Agglomeration: The incorporation of small clusters or attrition fragments into larger crystals has been included in many models describing Viedma ripening, as mentioned above. On a macroscopic scale this process is referred to as agglomeration. In agglomeration two particles (of any size) can collide with each other and, if a crystalline bridge between them is formed through crystal growth, a stable agglomerate is obtained. The mechanism therefore generally depends on the fluid dynamics, the size of the involved particles and the supersaturation. The latter influences whether a stable crystalline bridge forms in the time in which two particles are in the vicinity of each other.

In the context of Viedma ripening, it has been argued that small fragments formed by attrition/breakage can later undergo agglomeration. These agglomerates would undergo Ostwald ripening to a reduced extent, because the size of the agglomerate is larger than of the fragments. Experimental evidence suggests that agglomeration occurs in an enantioselective 
(A)

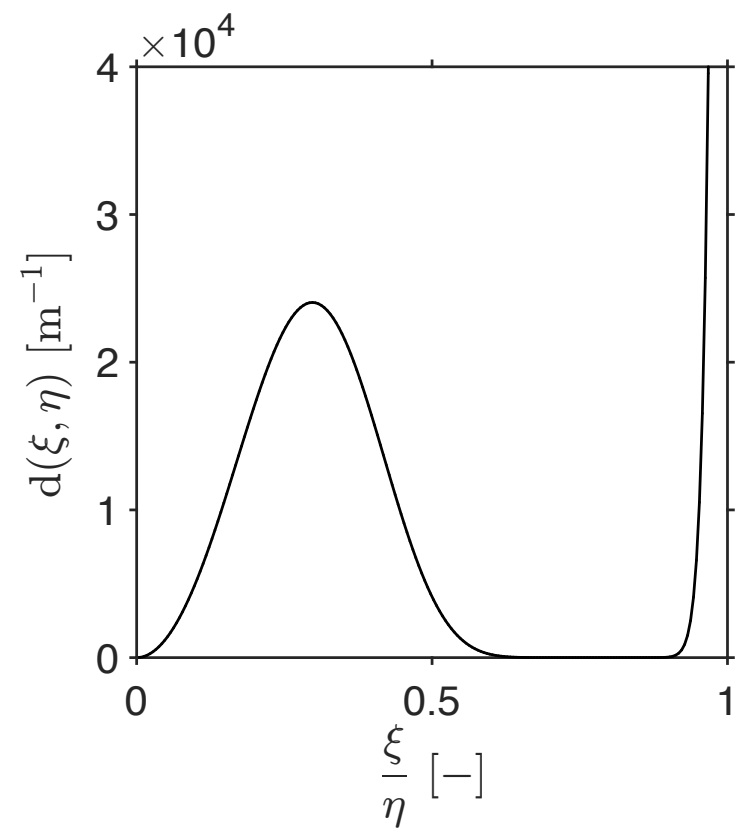

(B)

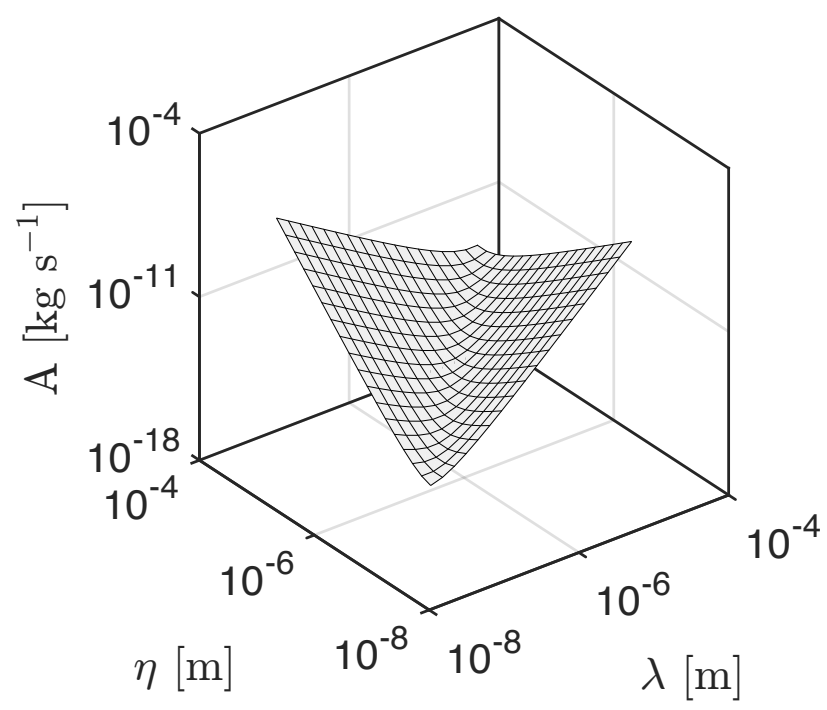

Figure 6: Distribution functions used in this work to describe breakage/attrition and agglomeration: (A) Daughter distribution $d(\lambda, \eta)$ (Eq. (4)) with $q=6$ and (B) Agglomeration kernel $A(\lambda, \eta)$ (Eq. (7)) with kinetic parameters $a_{1}=10^{20} \mathrm{~m}^{-2}$ and $a_{2}=10^{15} \mathrm{~s}^{-1}$.

way ${ }^{28}$ i.e., that crystals of the same handedness agglomerate preferentially with each other. Combined with the fact that agglomeration requires two particles, it is evident that crystals of the major enantiomer will on average undergo more agglomeration events than crystals of the minor enantiomer. This positive feedback loop is one of the reasons for the autocatalytic nature of Viedma ripening observed experimentally.

Although agglomeration models including shape effects have recently been developed ${ }^{48,49}$ the use of these models is beyond the scope of this work and hence agglomeration is expressed as a shape-conserving mechanism, i.e., we assume that the original particles and the resulting agglomerate exhibit the same shape and can hence be described using a single characteristic size. However, we do account for differences in collision rates (and hence agglomeration rates) between particles of unequal size by using a size-dependent agglomeration kernel introduced in earlier works ${ }^{37,50}$ :

$$
A(\lambda, \eta)=\left(\frac{\lambda+\eta}{2}\right)^{3} \frac{a_{2}}{1+a_{1} g(\lambda, \eta)}
$$


where $A(\lambda, \eta)$ is the agglomeration kernel expressing the number of agglomeration events per time between particles with characteristic lengths $\lambda$ and $\eta$. In Eq. (7) $a_{1}$ and $a_{2}$ in $A$, and $g(\lambda, \eta)$ is defined as

$$
g(\lambda, \eta)=\frac{(\lambda \eta)^{2}}{\lambda^{2}+\eta^{2}-\lambda \eta}
$$

In formulating the agglomeration kernel in this way, we have also assumed that agglomeration is volume- and therefore mass-conserving, i.e., that particles of sizes $\lambda$ and $\eta$ form a new particle of size $L=\sqrt[3]{\lambda^{3}+\eta^{3}} \cdot{ }^{50,51}$ In Figure 6B the agglomeration kernel is shown with the parameters used in this work. One can see that the kernel is symmetrical around the $\lambda=\eta$ line and indicates that smaller particles agglomerate more often with large particles which is largely driven by a higher probability of collision. Contrary to the mechanistic description of the agglomeration process above, we have also assumed that agglomeration is independent of supersaturation. Making this assumption is reasonable, because we expect low supersaturations to be present in all steady states in our processing schemes and hence the contribution of supersaturation to the agglomeration kernel can be treated as constant. Furthermore, this reduces the computational burden of the overall model tremendously, because the agglomeration kernel becomes time-invariant.

Nucleation: Primary nucleation, i.e., particle formation that is independent of the presence of already existing crystals of the same crystal form, is not explicitly included in the process model in this work for two main reasons. First, in stirred tank crystallizers with a substantial amount of particles already present, secondary nucleation-rather than primary nucleation-is the dominating process forming new particles. ${ }^{52,53}$ Here, we have included secondary nucleation through attrition as detailed above. Second, as shown elsewhere, ${ }^{45}$ combining Ostwald ripening and primary nucleation in a single consistent process model leads to considerable complexities and the resulting models are computationally expensive to solve. 
Racemization reaction: The description of mechanisms is completed by describing the racemization that converts $D$ enantiomer molecules to $L$ enantiomer molecules and vice versa:

$$
D \underset{k_{\mathrm{DL}}}{\stackrel{k_{\mathrm{LD}}}{\leftrightarrows}} L
$$

For the sake of simplicity, we assume that both the forward and backward reaction are first order reactions and that they have the same reaction rate constant. The reaction rate $r$ can then be written as:

$$
r=k_{\mathrm{DL}} c_{\mathrm{D}}-k_{\mathrm{LD}} c_{\mathrm{L}}=k_{r}\left(c_{\mathrm{D}}-c_{\mathrm{L}}\right)
$$

Table 1: Overview of kinetic rate constants and material constants used in the process model

\begin{tabular}{l|l|l} 
symbol & value & description \\
\hline$a_{1}$ & $10^{20} \mathrm{~m}^{-2}$ & const. in the agglomeration kernel \\
$a_{2}$ & $10^{15} \mathrm{~s}^{-1}$ & const. in the agglomeration kernel \\
$k_{\mathrm{a}}$ & $\pi$ & surface shape factor \\
$k_{\mathrm{b}, 1}$ & $0.1 \mathrm{~s}^{-1}$ & breakage rate const. \\
$k_{\mathrm{b}, 2}$ & 1 & breakage rate const. \\
$k_{\mathrm{g}}$ & $10^{-6} \mathrm{~m} \mathrm{~s}^{-1}$ & kinetic parameter in the growth rate \\
$k_{\mathrm{n}}$ & $1.568 \mathrm{~m}^{-1} \mathrm{~kg}^{-1}$ & const. in the seed population \\
$k_{\mathrm{r}}$ & $1 \mathrm{~s}^{-1}$ & racemization reaction kinetic parameter \\
$k_{\mathrm{v}}$ & $\pi / 6$ & volume shape factor \\
$M$ & $1.5013 \times 10^{-1} \mathrm{~kg} \mathrm{~mol}^{-1}$ & molar mass \\
$q$ & 6 & const. in the daughter distribution \\
$R$ & $8.3144 \mathrm{~J} \mathrm{~mol}^{-1} \mathrm{~K}^{-1}$ & gas constant \\
$V_{\mathrm{m}}$ & $9.57 \times 10^{-5} \mathrm{~m}^{3} \mathrm{~mol}^{-1}$ & molar volume \\
$\gamma$ & $2 \times 10^{-2} \mathrm{~J} \mathrm{~m}^{-2}$ & surface energy \\
$\rho_{\mathrm{c}}$ & $1.568 \times 10^{3} \mathrm{~kg} \mathrm{~m}^{-3}$ & crystal density
\end{tabular}

\section{PBE model and mass balances for the crystallizer and mill}

In order to evaluate the process flowsheets depicted in Figure 2, it is vital to describe the evolution of the particle size distributions of both enantiomers in the crystallizer and the mill. To this end, a population balance equation model ${ }^{54}$ will be used that incorporates the mechanistic descriptions of the key phenomena (as outlined above). For the general 
case depicted in the flowsheets above, two sets of two PBEs (one PBE per enantiomer and vessel) would have to be written for the crystallizer and the mill; however, for reasons of computational efficiency, we will treat the crystallizer-mill combination as a single processing unit in the following (which reduces the number of PBEs to solve to two; one for each enantiomer). This is equivalent to specifying that the flow rates between crystallizer and mill are kept constant in all case studies conducted later in this article. Adapting PBEs developed in previous work ${ }^{17,37}$ to the specific cases treated here, we can write:

$$
\begin{aligned}
\frac{\partial n_{i}(L, t)}{\partial t} & =-\frac{\partial\left(G\left(S_{i}, L\right) n_{i}(L, t)\right)}{\partial L}+ \\
& +\frac{L^{2}}{2} \int_{0}^{L} \frac{A\left(\sqrt[3]{L^{3}-\lambda^{3}}, \lambda\right) n_{i}\left(\sqrt[3]{L^{3}-\lambda^{3}}, t\right) n_{i}(\lambda, t)}{\left(L^{3}-\lambda^{3}\right)^{2 / 3}} \mathrm{~d} \lambda+ \\
& -n_{i}(L, t) \int_{0}^{\infty} A(L, \lambda) n_{i}(\lambda, t) d \lambda+ \\
& +\int_{L}^{\infty} K(\xi) d(\xi, \eta) n_{i}(\xi, t) \mathrm{d} \xi-K(\eta) n_{i}(\eta, t)+ \\
& +\frac{\dot{m}_{\mathrm{s}} n_{\mathrm{s}, i}(L)-\dot{m}_{c f} n_{i}(L, t)}{m_{\mathrm{c}}} \text { with } i \in\{\mathrm{D}, \mathrm{L}\}
\end{aligned}
$$

The left hand side of this equation represents the change of the number density distribution of particles of enantiomer $i, n_{i}(L, t)$, with characteristic size $L$ over time. The terms on the right hand side of the equation represent (in order of appearance): a convective term representing crystal growth and dissolution (and thus accounting for Ostwald ripening ${ }^{47}$ ), a term representing the formation of particles at size $L$ due to agglomeration of two smaller particles, the disappearance of particles of size $L$ due to agglomeration, a term representing particles that appear at size $L$ due to breakage of larger particles, a term that accounts for particles of size $L$ breaking into smaller fragments and, finally, a term representing the flow of particles into and out of the crystallizer-mill unit. The constitutive equations $(G, A, K$, and $d$ ) and their meaning were defined in the previous section, while the kinetic constants appearing in them were summarized in Table 1 . In the last term, $\dot{m}_{\mathrm{cf}}$ is the mass flow rate of the stream connecting the crystallizer-mill with the filtration unit, $\dot{m}_{\mathrm{s}}$, is the mass flow rate of the feed stream, $n_{\mathrm{s}, i}(L)$ is the size distribution of particles in the feed stream, and 
$m_{\mathrm{c}}$ represents the mass of suspension in the crystallizer-mill. For the solid-fed flowsheet (B in Figure 2) the particle size distribution in the feed stream will be defined in the results section, while in the liquid fed flowsheet (A in Figure 2), the feed stream is particle free, so that $n_{\mathrm{s}, i}(L)=0$.

A residence time, $\tau$, in the crystallizer-mill can be defined as

$$
\tau=\frac{m_{\mathrm{c}}}{\dot{m}_{\mathrm{cf}}}
$$

which is one of the key operating parameters that will be varied in the case studies. The description of the dynamics in the crystallizer-mill has to be completed with a mass balance for the liquid phase. In this mass balance the amount of solute molecules deposited/released onto/from the crystals through growth and dissolution, the racemization reaction and the flow terms must be accounted for; leading to: ${ }^{17,37}$

$\frac{\mathrm{d} c_{i}}{\mathrm{~d} t}=-3 k_{\mathrm{v}} \rho_{\mathrm{c}} \int_{0}^{\infty} G\left(S_{i}, L\right) L^{2} n_{i}(L, t) \mathrm{d} L-k_{\mathrm{r}}\left(c_{i}-c_{j}\right)+\frac{\dot{m}_{\mathrm{s}} c_{\mathrm{s}, i}+\dot{m}_{\mathrm{r}} c_{\mathrm{r}, i}-\dot{m}_{\mathrm{cf}} c_{i}}{m_{\mathrm{c}}}$ with $i, j \in\{L, D\}$ and $j \neq i$

where $\rho_{\mathrm{c}}$ is the crystal density, $c_{\mathrm{s}, i}$ is the concentration of enantiomer $i$ in the feed stream and $\dot{m}_{\mathrm{r}}$ and $c_{\mathrm{r}, i}$ are the mass flow rate and concentration of the stream being recycled to the crystallizer (that either originates from the solute concentrator (flowsheet A) or from the filtration unit (flowsheet B). The mass flow rate and concentration of this stream follows from the assumptions made about the filtration unit and solute concentrator, which are detailed in the following.

In order to solve the process model, the PBE is discretized using a first order finite difference scheme. The resulting system of ordinary differential equations (ODEs) is solved together with all mass balances in MatLAB using the built-in ODE solver ode15s. Details about the discretization procedure are provided in the supporting information. 


\section{Description of further processing units}

In this work the filtration unit is implemented following the assumption that the solid crystals in the crystallizer outlet stream are separated without loss of mother liquor (i.e., as dry solids). Similarly, the solute concentrator is modeled in a simplified way by assuming that it removes pure solvent from the filtrate stream. In our simulations the concentrator removes half of the solvent from the stream entering it."

In physical implementations of a filtration unit or a solute concentrator their performance will likely not follow such ideal behavior. Deviations from ideality would cause a reduction in yield and purity (filtration unit) or yield (solute concentrator). If significant levels of impurities are present in the feed, are generated during the process or if only part of the mother liquor is recycled for other reasons, a purge stream should be considered before the filtrate stream goes into the solute concentrator. However, for the sake of simplicity, we assume that purge streams are not necessary in this study. While these are obviously strong simplifications, our attention here is focused on the crystallizer-mill dynamics, which are decisive for both Viedma ripening and preferential crystallization and hence for enantiomeric purity. Furthermore, it would be rather straightforward to incorporate effects like residual cake wetness etc. in the respective mass balances.

\section{Results and discussion}

We will now use the process model detailed above to investigate how the solution and solidfed processes perform when different operating conditions are chosen. To this end, we will first investigate the start up behavior of the solution-fed flowsheet (flowsheet A, cf. Figure 2) and will identify stable steady states that can occur in it. Key operating conditions, such as the residence time of the crystallizer and the feed concentration, are then varied to iden-

\footnotetext{
"Note that the concentration of the solute in the remaining solvent should never exceed the solubility limit. To achieve this, the resulting concentrated stream could be at elevated temperature, e.g., when coming from the reboiler of a distillation column or a heated membrane module.
} 
tify regions at which such enantiopure steady states are attainable. Finally, we will also investigate the start up behavior and stable steady states in the solid-fed flowsheet B (cf. Figure 2).

\section{Start-up investigation of the solution-fed continuous process}

As a first case study the start-up behavior of the process shown in Figure 2A without recycle is investigated. In Table 2 the initial and process conditions are summarized for these case studies (labelled liq1 - liq5). The initial concentration in the solution phase is always set equal to the feed concentration, $c_{\mathrm{s}, i}=c_{0, i}$, while different enantiomeric excesses are provided in the solid phase. The particle size distributions (PSDs) of the seed crystals are given as:

$$
n_{0, i}(L)=k_{\mathrm{f}} \theta_{i} \exp \left(-\frac{(L-\chi)^{2}}{2 \sigma^{2}}\right), \text { with } i \in\{\mathrm{D}, \mathrm{L}\}
$$

where $\chi$ is the mean size of the distribution and $\sigma$ gives the standard deviation (broadness) of the distribution and the constant $k_{\mathrm{f}}$ is chosen such that a total seed mass (sum of both enantiomer populations) of $1.5684 \times 10^{-2} \mathrm{~kg}$ per $\mathrm{kg}$ of crystallizer content results. In this article we always used seed PSDs with $\chi=4 \times 10^{-5} \mathrm{~m}$ and $\sigma=1 \times 10^{-5} \mathrm{~m}$. The total seed mass is distributed on the two enantiomers, so that a specific initial enantiomeric excess in the solid phase, e.e.0, is obtained. The constant $\theta_{\mathrm{L}}=0.5\left(e . e_{0}+1\right)$ and $\theta_{\mathrm{D}}=1-\theta_{\mathrm{L}}$ is included in Eq. (14) for this purpose. The e.e in the solid phase can be calculated as:

$$
\text { e.e. }=\frac{\left(m_{\text {solids }, \mathrm{L}}-m_{\text {solids, } \mathrm{D}}\right)}{\left(m_{\mathrm{solids}, \mathrm{D}}+m_{\mathrm{solids}, \mathrm{L}}\right)}
$$

where the crystal masses for the L and D enantiomer can easily be obtained from the corresponding PSD at any given time.

Starting with study liq1 as our base case, we show the evolution of the (volume-weighted) PSDs for both enantiomers, the enantiomeric excess in the solid phase, as well as the mass of crystals of both enantiomers in Figure 7. Focusing our attention first on the left hand panel 
Table 2: Conditions for the parameter study of the solution-fed process.

\begin{tabular}{c|c|c|c|c|} 
study & $\tau[\mathrm{h}]$ & $c_{\mathrm{s}, i}\left[\mathrm{~g} \mathrm{~kg}^{-1}\right]$ & recycle $[\%]$ & e.e.0 $[-]$ \\
\hline liq1 & 4 & 45 & 0 & 0.1 \\
liq2 & 4 & 45 & 0 & $\{0.025,0.05,0.1,0.2,0.3,0.5\}$ \\
liq3 & 4 & $\{35,40, \ldots, 60\}$ & 0 & 0.1 \\
liq4 & $\{1,1.5, \ldots, 5\}$ & $\{35,40, \ldots, 70\}$ & 0 & 0.1 \\
liq5 & $1-5$ & $\{35,40, \ldots, 70\}$ & $\{25,50,75,100\}$ & 0.1
\end{tabular}

of this figure, where the evolution of the volume-weighted PSD of the L enantiomer is shown, we quickly see that the seed particles are rapidly milled down to smaller sizes. This peak at smaller sizes ultimately stabilizes at a constant value, indicating that a steady state is reached. Conversely, in the right hand panel, the evolution of the PSD of the D enantiomer is shown. Similarly to the L enantiomer, we see that the D enantiomer seed particles are also milled down rapidly, but in contrast to the $\mathrm{L}$ enantiomer, there are no crystals left of the D enantiomer after some time. This indicates that the operating conditions selected for the base case lead to an enantiopure steady state. This is confirmed in the middle panel of Figure 7, where the evolution of the e.e., as well as the mass of $\mathrm{L}$ and $\mathrm{D}$ crystals is shown. Since the starting condition of the liquid phase is supersaturated ${ }^{* *}$, there is initially an increase in the crystal mass for both enantiomers. Subsequently, the crystalline mass of both enantiomers is decreasing, but the initially enriched L enantiomer recovers and reaches a stable level, while the mass of D crystals is continuously dropping until there are ultimately no crystals of the D enantiomer present at the steady state. The evolution of the e.e. in the center panel reflects this behavior as well and shows that an enantiopure steady state (in the solid phase) is reached under the operating conditions chosen for study liq1.

Our second set of simulations, liq2, is aimed at showing the effect of the initial e.e.0 on the start up. To this end, the e.e.0 is varied while keeping all other conditions the same as in the previously discussed case (liq1). To fingerprint the dynamics of the process, we report the evolution of the enantiomeric excess in the solid phase in Figure 8A. One quickly sees that an enantiopure steady state is reached as long as there is an enrichment

** The solubility at racemic conditions is $31.8 \mathrm{~g} \mathrm{~kg}^{-1}$. 

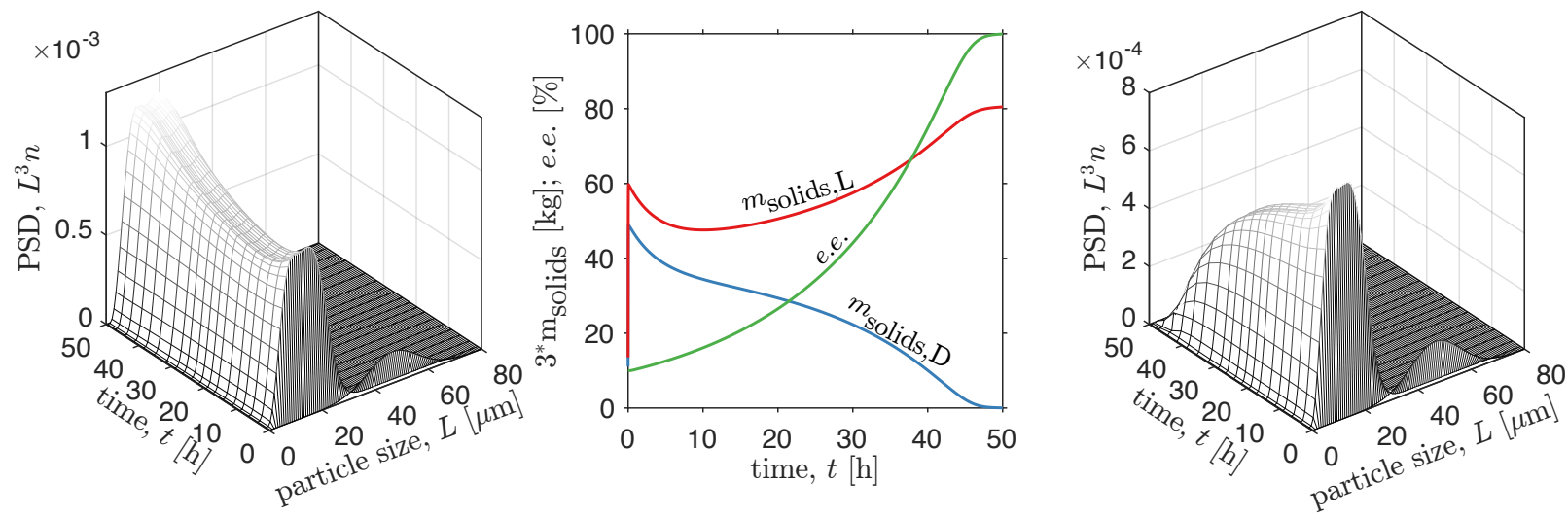

Figure 7: Exemplary evolution of particle size distributions over time for a simulation with enantiopure steady state (study liq1 in Table 2). The L-enantiomer (left) increases while the D-enantiomer decreases (right). The mass of the solids in the crystallizer and the enantiomeric excess are drawn vs. time in the middle.

(i.e., e.e.o $\in[-1 ; 1] \backslash\{0\})$. Furthermore, all cases reach the same enantiopure steady state (same particle size, concentrations, etc.). While not explicitly shown, the thermodynamics and kinetics of both enantiomers are identical, so that any enrichment in the D enantiomer would lead to an enantiopure steady state with D crystals present. From this figure, we also see that larger enrichments lead to shorter times to steady state, as one might expect. In Figure 8B, we show the solid e.e. evolution for feed concentrations ranging from $35-$ $60 \mathrm{~g} \mathrm{~kg}^{-1}$ (simulations liq3 in Table 2).

From this figure, we observe that higher feed concentrations result in longer times to steady state. Furthermore, we observe that we do not achieve enrichment anymore when exceeding a maximum feed concentration, but rather converge towards a racemic steady state. In study liq3 the maximum feed concentration leading to an enantiopure steady state lies between 55 and $60 \mathrm{~g} \mathrm{~kg}^{-1}$, which can be deduced by considering the profiles for a feed concentration of $55 \mathrm{~g} \mathrm{~kg}^{-1}$, which is trending upwards, and for feed concentration of $60 \mathrm{~g} \mathrm{~kg}^{-1}$, which is trending downwards to a racemic state. Indeed, when running these simulations until they reach steady state, an e.e. of 1 and 0 is ultimately reached, respectively. This behavior echoes earlier studies carried out on preferential crystallization processes without the presence of a racemization reaction in the liquid phase. ${ }^{17}$ 
(A)

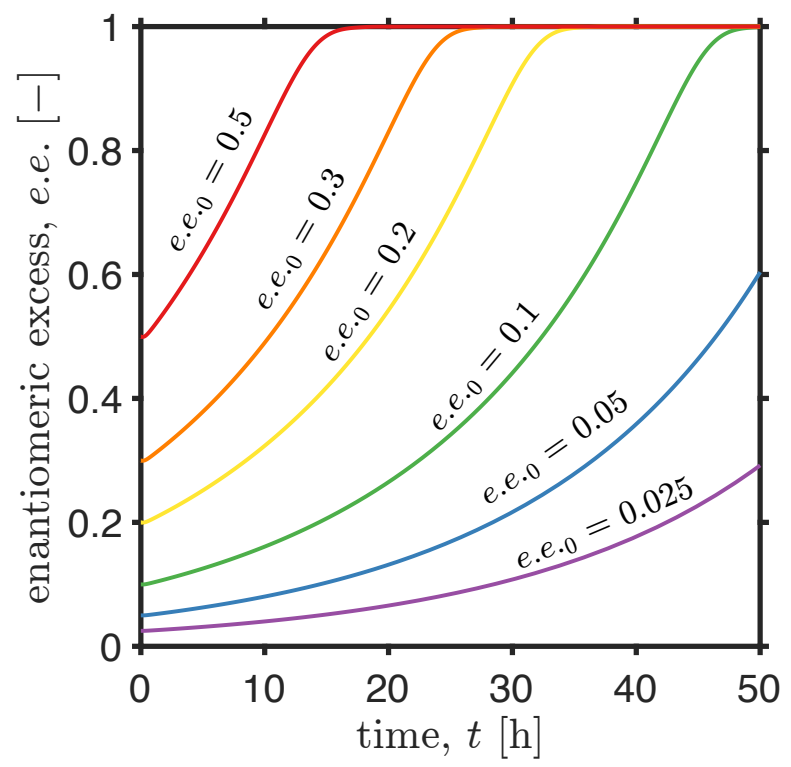

(B)

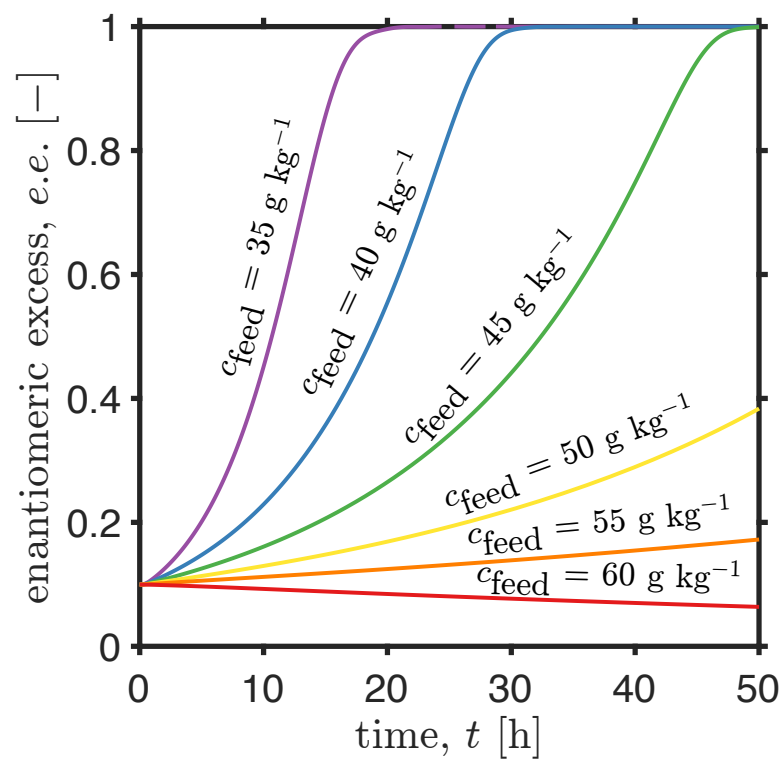

Figure 8: Start up behavior of the liquid-fed process: Evolution of enantiomeric excess in the solid phase when (A) starting from different initial enantiomeric excesses (e.e.0), and (B) supplying different feed concentrations. In both cases the crystallizer residence time is $\tau=4 \mathrm{~h}$; all operating conditions are given as liq2 and liq3, respectively, in Table 2 .

\section{Steady state investigation of the solution-fed continuous process}

We now investigate how the binary steady state behavior observed for the liquid-fed flowsheet (flowsheet A in Figure 2) changes when the residence time of the crystallizer is varied as well. For this, an extensive parameter study (72 simulations in total, cf. liq4 in Table 2) is carried out where for each set of parameters the simulation is run until steady state is reached. The steady state enantiomeric excesses in the solid phase again showed the binary behavior (100\% enantiopurity vs. racemic) regardless of the residence time and feed concentration chosen, i.e., no intermediate enantiomeric enrichment at steady state was detected in any of the simulations carried out. The data are reported in Figure 9, where operating conditions leading to enantiopure steady states are shown in red, while the racemic steady states are reported in blue. The region where no simulation data is available is reported in gray. One can see that there is a sizeable region where enantiopurity can be reached. At higher residence times, the maximum feed concentration, at which the process 
maintains enantiopurity, increases. It is clear that longer residence times at the same feed concentration mean a lower steady state concentration and thus potentially higher yield, however, the increase in residence time might not be offset by this increase in yield, so that the productivity (mass of enantiopure crystals per time and per mass of crystallizer content) could be decreasing. In order to optimize the productivity of the process, the tradeoff between residence time and feed concentration would have to be analyzed.

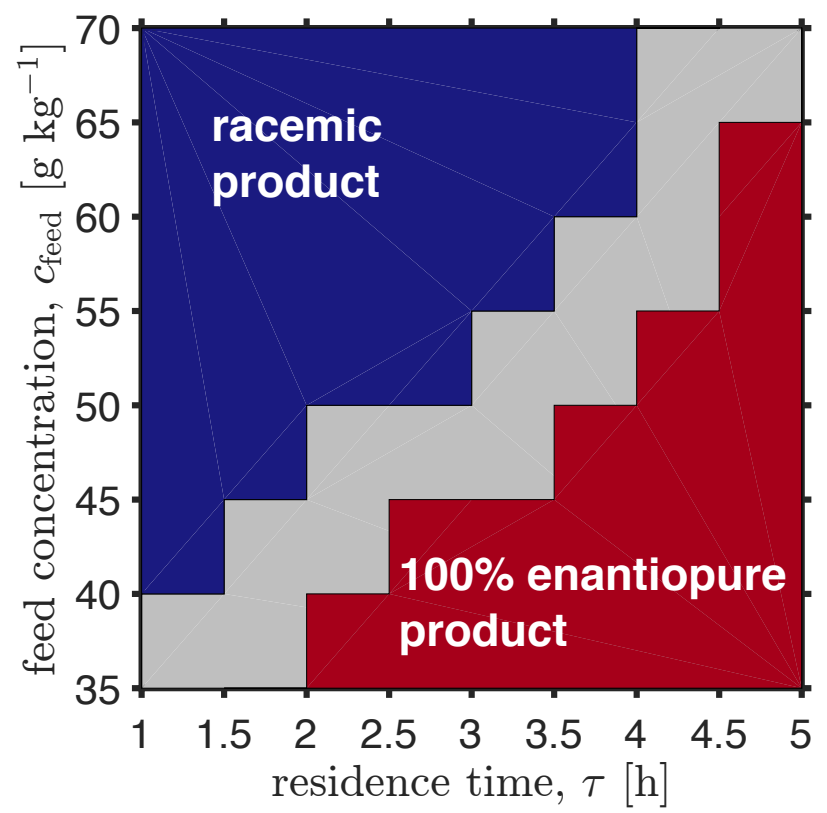

Figure 9: Steady state behavior of the liquid-fed process: steady state enantiomeric excesses are reported for different feed concentrations and residence times (study liq4). Enantiopure steady-states are shown in red, racemic steady-states in blue. In the gray area no simulations were carried out and the steady state outcome is undetermined.

In the cases so far, flowsheet $\mathrm{A}$ has been operated without any recycle of the mother liquor. Purging the complete mother liquor from the process is wasteful, because valuable product, as well as racemization catalyst (as well as solvent) are lost. Rather than optimizing the productivity for the flowsheet without recycle, we focus on the effect of recycling (part of) the mother liquor in the following. To this end, we include the recycle part of flowsheet A (drawn as the grayed out part in Figure 2A). In this configuration, (part of) the mother liquor from the filtration unit will be recycled back into the crystallizer in order to improve the yield of the overall process and arrive at an intensified configuration. Due to impurity 
accumulation a part of the recycle stream might have to be purged out from the process. While we assume here that no impurities are present, in a real scenario, such considerations need to be kept in mind. In the following, we recycle $25 \%, 50 \%, 75 \%$ and $100 \%$ of the mother liquor stream, while the remainder would be removed as a purge stream (i.e., 75\%, 50\%, 25\% and no purge, respectively). The conditions are summarized as study liq5 in Table 2. The remaining mother liquor stream passes through the solute concentrator, where pure solvent is removed without loss of product or racemization catalyst. In Figure 10 we report the results for all four recycle ratios varying the feed concentration again from 35 to $70 \mathrm{~g} \mathrm{~kg}^{-1}$ and the residence time from 1 to $5 \mathrm{~h}$ (in total 288 additional simulations).

The main finding from this study is that higher recycle streams shrink the operating space where enantiopurity is reached at steady state. In terms of the shape of these regions, the behavior found in study liq4 is closely mirrored in the liq5 studies, i.e., lower feed concentrations and higher residence times are beneficial to reach enantiopure steady states. However, the major take away message from study liq5 is that the proposed process can deliver a theoretical yield of $100 \%$ at full enantiomeric purity (by recycling $100 \%$ of the mother liquor) and that the operating region for this seems to be sizeable.

\section{Solid-fed continuous process}

In this section we investigate the steady state behavior of the solid-fed process (as drawn in Figure 2B). This has the advantage that the filtrate could be recycled directly, i.e. additional work-up of the solute is not necessary, because the solvent amount in the system remains constant. As far as we know, no investigation of such a continuous Viedma ripening process has been carried out in the literature yet. Thus, we aim at determining if such a process, carried out in a single MSMPRC, can reach enantiopurity. To investigate this, we again conduct parameter studies, which are listed in Table 3. With respect to the size distribution of the particles used in the feed stream, we use the same distribution (Eq. (14)) with the same mean size $\left(\chi=4 \times 10^{-5} \mathrm{~m}\right)$ and broadness $\left(\sigma=1 \times 10^{-5} \mathrm{~m}\right)$ as before, but specify 

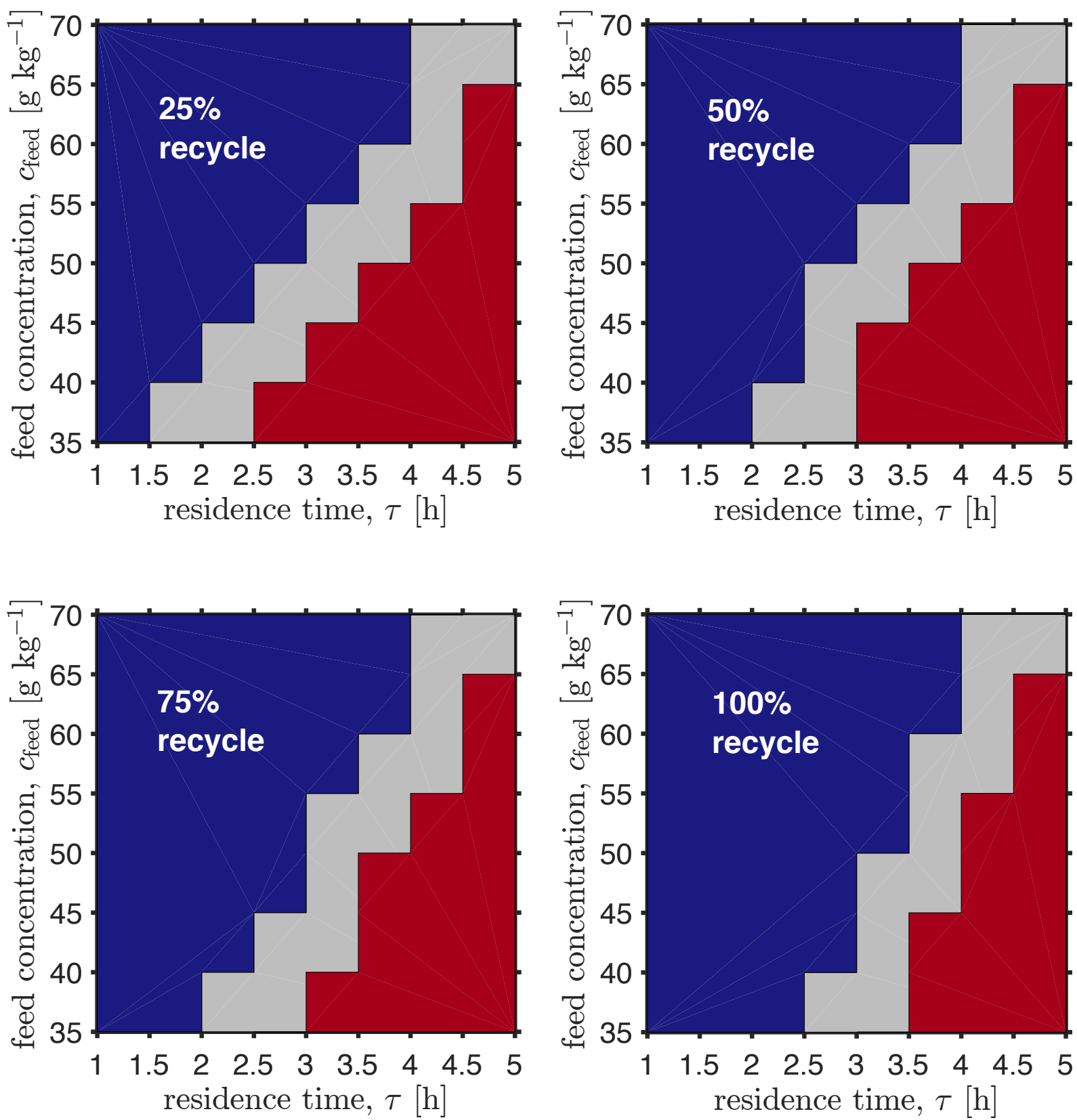

Figure 10: Steady state behavior of the liquid-fed process with mother liquor recycle: steady state enantiomeric excesses obtained for the operating conditions summarized as study liq5 in Table 2 are reported. Each panel represents a different fraction of mother liquor recycle to the crystallizer $(25 \%, 50 \%, 75 \%, 100 \%)$. Enantiopure steady-states are shown in red, racemic steady-states in blue. In the gray area no simulations were carried out and the steady state outcome is undetermined.

that the same mass of solids of the $\mathrm{D}$ and $\mathrm{L}$ enantiomer is fed. The initial e.e.0 is now set to 0.5 , in an attempt to shorten the time required until steady state is reached. Study s1 is 
conducted to illustrate the start-up behavior of the process, and highlight the differences to the previous studies on the solution-fed process.

Table 3: Parameter studies carried out on the solid-fed process.

\begin{tabular}{c|c|c|c|c} 
study & $\tau[\mathrm{h}]$ & $c_{0}\left[\mathrm{~kg} \mathrm{~m}^{-3}\right]$ & $\dot{m}_{\text {feed }}\left[\mathrm{kg} \mathrm{h}^{-1}\right]$ & $e . e_{\cdot 0}[-]$ \\
\hline $\mathrm{s} 1$ & $\{5,10, \ldots, 25\}$ & 35 & 5 & 0.5 \\
$\mathrm{~s} 2$ & $\{5,6.25, \ldots, 25\}$ & 35 & $\{1,2, \ldots, 10\}$ & 0.5
\end{tabular}

The results of the start-up study s1 are shown in Figure 11 and reveal that the steady state arrives at intermittent enantiomeric excesses, which is in stark contrast to the solution-fed process considered previously. Even for long residence times of $25 \mathrm{~h}$, steady states beyond an e.e. of 0.9 seem hard to reach. The steady state e.e. plateaus out with higher residence times, not increasing (or decreasing) anymore. Note that all simulations were run until the slope of the e.e. vs. time curve was negligibly small and some were run for even longer process times, so that the reported values represent true steady states. From these initial observations one can already see that reaching full enantiopurity in a continuous Viedma ripening process carried out in a single MSMPRC is not feasible, because the continued addition of already existing crystals of the counter enantiomer and the Viedma ripening kinetics are ill-suited for a continuous process.

To investigate this in more depth and to gain an understanding of how the feed rate affects the steady state outcome of the solid-fed process, study s2 is conducted. Study s2 comprises residence times from 5 to $25 \mathrm{~h}$ and solid feed rates of 1 to $10 \mathrm{~kg} \mathrm{~h}^{-1}$. The residence time was increased in steps of $1.25 \mathrm{~h}$ and the solid feed flow was increased in steps of $1 \mathrm{~kg} \mathrm{~h}^{-1}$. Therewith in total the results of 170 simulations are shown in Figure 12. From this figure one gains an appreciation that an increase in solid feed rate slightly improves the steady state e.e. at a constant residence time, while the aforementioned plateuing effect observed in study s1 is observed regardless of the solid feed rate, i.e., full enantiopurity was never reached in the continuous Viedma ripening process.

Analyzing the map shown in Figure 12 in more detail, we see that the residence time 


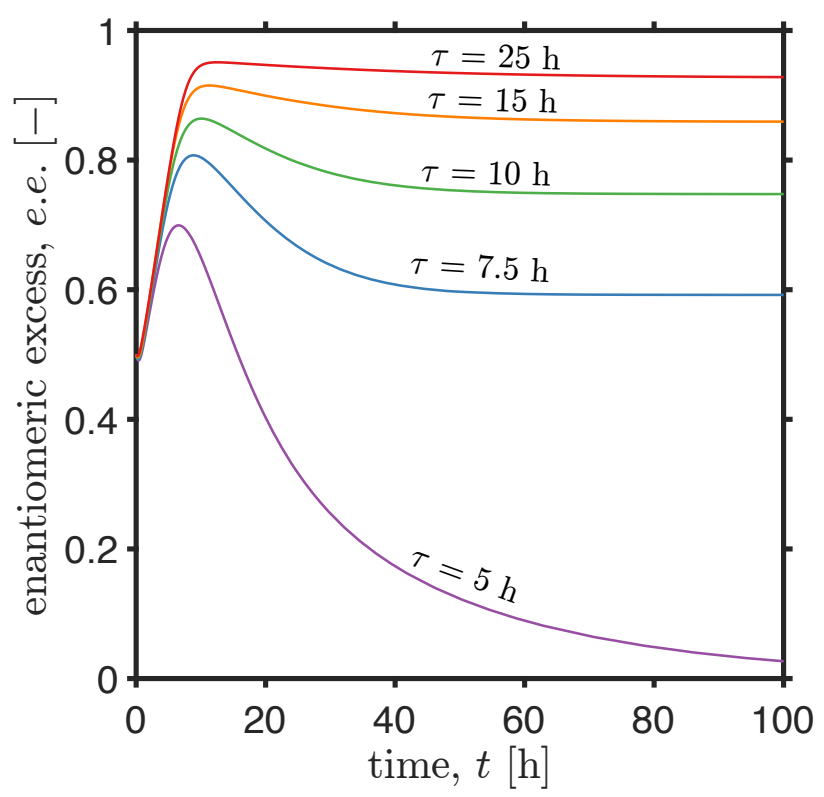

Figure 11: Start-up behavior of the solid-fed process: enantiomeric excess vs. time is shown for different residence times (study s1 in Table 3).

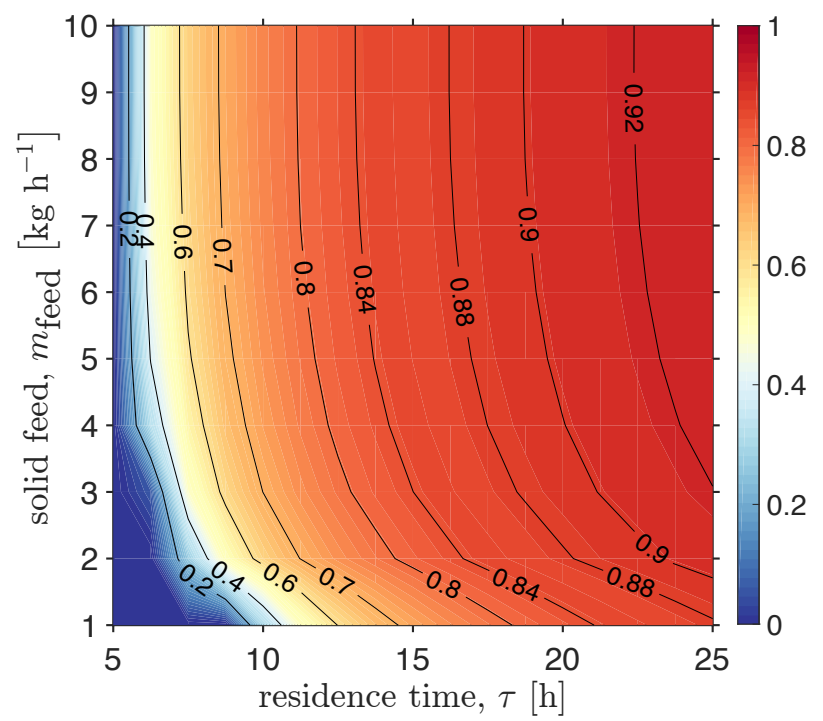

Figure 12: Steady state behavior of the solid-fed process: The map shows the solid state e.e for the investigated residence times and associated solid feed rates (study s2). The solid e.e. varies from 0 to 0.92 and is depicted with the color scale ranging from racemic (dark blue) to enantiopure (dark red) drawn on the right hand side.

has a stronger effect on the solid state composition compared to the feed concentration. Reasonably enriched steady states are only found above residence times of 8 hours. Below 8 hours only slightly enriched (or completely racemic) solid product can be obtained from the 
process. The solid state purity enhances quickly to enantiomeric excesses of about 0.7 by increasing the residence time to roughly 12 hours. For higher residence times the product purity still increases, but as previously shown in Figure 11 there are clearly diminishing returns. It is noteworthy that the supersaturation levels attained at steady state for all simulations in studies s1 and s2 are close to equilibrium, so that all kinetics, even Ostwald ripening, are at play in these simulations.

\section{Discussion of assumptions, limitations and possible gener-}

\section{alizations}

In this section of our article we want to point out limitations as well as possible extensions and generalizations of the presented study. We highlight that the constitutive equations employed in this investigation were kept in the right order of magnitude for each of the phenomena considered (solubility, crystal growth/dissolution, breakage, agglomeration, etc.). However, they do not represent a specific model system and hence no absolute values should be extracted from our findings. Nonetheless, we believe that even qualitative trends are a worthwhile result that can steer eventual experimental investigations and that the simulations can act as a feasibility check. In addition, we believe that the model can be used to describe a crystallization process for a specific model substance once kinetic data has been established for it.

\section{Limitations and discussion of assumptions}

Racemization reaction: Our model equations are valid for liquid phase racemization reactions of any speed. The results reported here are for cases where the liquid phase reaction

is much faster than the crystallization kinetics. It has been shown both experimentally ${ }^{24,55}$ and theoretically ${ }^{37}$ that fast racemization kinetics are beneficial for Viedma ripening (faster attainment of complete deracemization in batch Viedma ripening). In the limit of no racem- 
ization reaction occurring we expect that Viedma ripening stops occurring. The preferential crystallization process however is still able to deliver enantiopure material, however at a reduced yield and productivity. Therefore, the study presented here should be seen as an ideal case.

Removal of solvent in the solvent removal unit: The maximum amount of solvent that can be removed in the solvent removal unit without adverse effects is limited by the solubility of the solute in the solvent and by general characteristics of the solvent and solute molecules. Namely, the solubility of the solute in the solvent should never be exceeded, as crystallization and fouling would otherwise occur in the solute concentrator. The solute concentrator could be a distillation column or a membrane system where the pure solvent is selectively removed. In the case of distillation, the order of the boiling points, e.g., of impurities, racemization catalyst, solvent and solute molecules should be considered, as well as the thermal stability of all molecules and the racemization catalyst itself in the process. Thermal stability is, for instance, a key issue when using enzymes as racemization catalysts. Furthermore, in our implementation of these processing units, we have assumed that no racemization catalyst is removed (lost) in the solute concentrator, i.e., we have assumed that the processing unit is highly selective. If this is not attainable, one would have to continuously replenish the racemization catalyst.

Crystal characteristics: A limitation of this study is that the crystals have been considered to be well described by a single characteristic size. If there is a wide variety of shapes occuring in the crystallizer (and when the encountered shapes are dependent on the process parameters, e.g., the supersaturation), then our model can only be considered approximate. The key characteristic of the substance is mainly captured in the growth rate as a function of the temperature and the crystal size. Due to the implementation of the size dependency into the growth rate crystal characteristics such as the crystal density and surface energy are also incorporated. 
Breakage: The equations describing breakage in our model are the daughter distribution and the breakage rate. Both equations include parameters, where assumptions had to be taken. Even though the equations describing these have already been used in previous studies, ${ }^{17,37}$ especially the breakage rate and correspondingly the minimum particle size that can be reached for a given combination of mill, substance and operating conditions, are decisive for the kinetics of Viedma ripening (as also shown in Iggland and Mazzotti ${ }^{37}$ ). While our model allows for a qualitative analysis of process outcomes, a quantitative prediction of process performance would require establishing kinetics through experiments on the given combination of mill, crystals and operating conditions.

Nucleation: Due to low supersaturation levels at steady state when milling operations are used, we chose not to implement primary nucleation as a mechanism into the process model. If necessary, i.e., in the case of a solute/solvent combination leading to fast nucleation kinetics, primary nucleation could be incorporated into a consistent model together with Ostwald ripening (and thus Viedma ripening) by using kinetic rate equation models. ${ }^{45}$ However, this would lead to a computationally more demanding model.

\section{Generalization of the processing concepts}

We would like to point out that the "racemization reaction" can be any reversible reaction that forms/destroys a chiral center. This includes bimolecular reactions where achiral reactants are combined into a chiral product. The reaction itself does not need to exhibit stereospecificity. For batch Viedma ripening, an example of a Michael addition run concomitantly with the crystallization of a conglomerate forming chiral product has been recently reported in the literature ${ }^{41}$. It is noteworthy however, that the reactants should exhibit a higher solubility than the product molecule as otherwise a mixture of crystals of the reactants and a chirally pure product may be obtained from a continuous process, hence impacting product purity. While the processes presented here can only be applied to conglomerate 
forming systems, racemic compound forming molecules can sometimes be functionalized, such that the functionalized molecules crystallize as a conglomerate; an example of this has been shown by Noorduin et al. ${ }^{26}$ for naproxen (which crystallizes as a racemic compound) and naproxen-methyl/ethylester (which crystallize as conglomerates). The possibility to form a salt or co-crystals that crystallize as conglomerates has also been reported ${ }^{56}$.

\section{Summary and conclusions}

Using a thorough mathematical model we investigated two novel continuous crystallization processing flowsheets. We would again like to highlight that the study conducted is a qualitative one, because we relied on order of magnitude estimations for the kinetic parameters in our model. Therefore, no quantitative conclusions should be drawn from our results. However, the model used in our study is also one that is based on a mechanistic description of the individual phenomena, so that qualitative conclusions drawn can be expected to be valid. Furthermore, if kinetics are indeed available for a given combination of enantiomers, solvent and processing equipment, they can be implemented in the process model, so that quantitatively meaningful results are obtained.

As far as qualitative results go, the first, liquid-fed flowsheet (a continuous preferential crystallization process) achieves either the complete separation of enantiomers of a conglomerate forming system using a single MSMPRC with attached suspension mill or it delivers a racemic product, depending on the choice of operating parameters. In this context it is striking that no intermediate outcomes are realizable. Furthermore, we have shown that by combining the MSMPRC-mill combination with ancillary units for solvent-removal (such as distillation or a membrane unit), the overall process is able to achieve enantiopurity at a theoretical yield of $100 \%$. In that configuration, the presence of a racemization reaction in solution effectively allows not only to separate enantiomers, but also to convert the undesired enantiomer into the desired one. Thus, the ability to racemize enantiomers in solution 
essentially allows doubling the overall yield in comparison to cases where no racemization is possible. ${ }^{17}$ Through a parametric analysis we have shown that there is a considerable operating space where enantiopurity and high (or complete) yields can be achieved and we have highlighted the qualitative link between key operating parameters, such as the residence time and feed concentration, and the process outcome. In contrast, the second, solid-fed flowsheet (a continuous Viedma ripening process) can reach an enrichment of roughly $90 \%$ e.e., but requires excessively long residence times with correspondingly low productivities to achieve this. We have again elucidated how the process outcome qualitatively depends on the main operating parameters of the process, namely the residence time and the solid feed rate to the crystallizer. While we have shown the existence of a tradeoff between productivity and enantiopurity for both the liquid- and solid-fed crystallization processes, we have not quantified this tradeoff here as we deemed this to lie outside the scope of the present work.

If higher steady state enantiomeric excesses are desired from a solid-fed crystallization process with reasonable productivities, more processing equipment (more than a single MSMPRC) would be required to design enhanced processes. For instance, temperature cycles were recently shown to be highly effective in achieving deracemization in batch Viedma ripening ${ }^{57}$. The same principle could be implemented in a continuous process using a second vessel at elevated temperature - essentially a dissolution tank - connected to the crystallizer in a loop. ${ }^{58}$ While the overall process achieves higher steady state enantiomeric excess, the productivity of such an operation has not yet been studied and it comes at an increased capital cost.

The study presented here also serves as a reminder that a batch vs. continuous processing decision should be considered on a case by case basis. The continuous preferential crystallization process including a racemization reaction can outperform its batch counterparts (see also Vetter et al. ${ }^{17}$ for more case studies), and on top of that it is able to recover from process disturbances (such as the sudden occurrence of crystals of the undesired enantiomer), because it runs at a stable steady state. Its batch counterpart, always striving towards the 
thermodynamic equilibrium, would suffer from such accidental seeding and would deliver an enantiomerically impure product. In contrast, in a batch Viedma ripening process an initially enriched solid phase always develops to an enantiopure product in a thermodynamically stable fashion (if enough time is allotted to the process), while our results for the continuous Viedma ripening process indicate that full enantiomeric purity is exceedingly hard to obtain in such a process. A prudent designer of an enantiomer separation process is therefore well-advised to base a decision for separation strategy and technology not on a "one size fits all" approach, but rather on fundamental insights.

\section{Acknowledgement}

TK thanks The University of Manchester for providing a PhD scholarship. Further thanks to Professor Roger Davey for stimulating discussions.

\section{Supporting Information Available}

Discretized model equations, their numerical implementation, nomenclature/units, and more detailed results of the simulations can be found in the supporting material. This material is available free of charge via the Internet at http://pubs.acs.org/.

\section{References}

(1) Eriksson, T.; Bjöurkman, S.; Roth, B.; Fyge, A.; Höuglund, P. Stereospecific determination, chiral inversion in vitro and pharmacokinetics in humans of the enantiomers of thalidomide. Chirality 1995, 7, 44-52.

(2) Eleutherakis-Papaiakovou, V.; Bamias, A.; Dimopoulos, M. A. Thalidomide in cancer medicine. Ann. Oncol. 2004, 15, 1151-1160. 
(3) Maier, N. M.; Franco, P.; Lindner, W. Separation of enantiomers: needs, challenges, perspectives. J. Chromatogr. A 2001, 906, 3-33.

(4) Rajendran, A.; Paredes, G.; Mazzotti, M. Simulated moving bed chromatography for the separation of enantiomers. J. Chromatogr. A 2009, 1216, 709-738.

(5) Kozma, D. CRC handbook of optical resolutions via diastereomeric salt formation; CRC Press: Boca Raton, FL, 2001.

(6) Jacques, J.; Collet, A.; Wilen, S. H. Enantiomers, racemates, and resolutions; Wiley: Hoboken, NJ, 1981.

(7) Springuel, G.; Leyssens, T. Innovative chiral resolution using enantiospecific cocrystallization in solution. Cryst. Growth Des. 2012, 12, 3374-3378.

(8) Wang, Z.; Cai, C.; Lin, Y.; Bian, Y.; Guo, H.; Chen, X. Enantioselective separation of ketoconazole enantiomers by membrane extraction. Sep. Purif. Technol. 2011, 79, $63-71$.

(9) Lorenz, H.; Seidel-Morgenstern, A. Processes to separate enantiomers. Angew. Chem. Int. Ed. 2014, 53, 1218-1250.

(10) Elsner, M. P.; Menéndez, D. F.; Muslera, E. A.; Seidel-Morgenstern, A. Continuous Separation of Isomers in Fluidized Bed Crystallizers. Cryst. Growth Des. 2016, 16, $1409-1419$.

(11) Sakai, K.; Hirayama, N.; Tamura, R. Novel Optical Resolution Techniques; Springer: New York City, NY, 2007.

(12) Elsner, M. P.; Ziomek, G.; Seidel-Morgenstern, A. Simultaneous preferential crystallization in a coupled, batch operation mode-Part I: Theoretical analysis and optimization. Chem. Eng. Sci. 2007, 62, 4760-4769. 
(13) Elsner, M. P.; Ziomek, G.; Seidel-Morgenstern, A. Efficient separation of enantiomers by preferential crystallization in two coupled vessels. AIChE J. 2009, 55, 640-649.

(14) Galan, K.; Eicke, M. J.; Elsner, M. P.; Lorenz, H.; Seidel-Morgenstern, A. Continuous Preferential Crystallization of chiral molecules in single and coupled Mixed-Suspension Mixed-Product-Removal crystallizers. Cryst. Growth Des. 2015, 15, 1808-1818.

(15) Qamar, S.; Elsner, M. P.; Hussain, I.; Seidel-Morgenstern, A. Seeding strategies and residence time characteristics of continuous preferential crystallization. Chem. Eng. Sci. 2012, $71,5-17$.

(16) Qamar, S.; Galan, K.; Elsner, M. P.; Hussain, I.; Seidel-Morgenstern, A. Theoretical investigation of simultaneous continuous preferential crystallization in a coupled mode. Chem. Eng. Sci. 2013, 98, 25-39.

(17) Vetter, T.; Burcham, C. L.; Doherty, M. F. Separation of conglomerate forming enantiomers using a novel continuous preferential crystallization process. AIChE J. 2015, $61,2810-2823$.

(18) Würges, K.; Petruševska-Seebach, K.; Elsner, M. P.; Lütz, S. Enzyme-assisted physicochemical enantioseparation processes-Part III: Overcoming yield limitations by dynamic kinetic resolution of asparagine via preferential crystallization and enzymatic racemization. Biotechnol. Bioeng. 2009, 104, 1235-1239.

(19) Binev, D.; Seidel-Morgenstern, A.; Lorenz, H. Experimental study and simplified mathematical description of preferential crystallization. Chirality 2005, 17, S183-S195.

(20) Kondepudi, D. K.; Kaufman, R. J.; Singh, N. Chiral symmetry breaking in sodium chlorate crystallizaton. Science 1990, 250, 975-976.

(21) Viedma, C. Chiral symmetry breaking during crystallization: complete chiral purity induced by nonlinear autocatalysis and recycling. Phys. Rev. Lett. 2005, 94, 065504. 
(22) Viedma, C. Chiral symmetry breaking and complete chiral purity by thermodynamickinetic feedback near equilibrium: implications for the origin of biochirality. Astrobiology 2007, 7, 312-319.

(23) Cheung, P. M. Complete asymmetric amplification of ethylenediammonium sulfate using an abrasion/grinding technique. Chem. Commun. 2008, 987-989.

(24) Noorduin, W. L.; Izumi, T.; Millemaggi, A.; Leeman, M.; Meekes, H.; Van Enckevort, W. J. P.; Kellogg, R. M.; Kaptein, B.; Vlieg, E.; Blackmond, D. G. Emergence of a single solid chiral state from a nearly racemic amino acid derivative. J. Am. Chem. Soc. 2008, 130, 1158-1159.

(25) Noorduin, W. L.; Meekes, H.; Bode, A. A. C.; van Enckevort, W. J. P.; Kaptein, B.; Kellogg, R. M.; Vlieg, E. Explanation for the emergence of a single chiral solid state during attrition-enhanced Ostwald ripening: survival of the fittest. Cryst. Growth Des. 2008, 8, 1675-1681.

(26) Noorduin, W. L.; Kaptein, B.; Meekes, H.; van Enckevort, W. J. P.; Kellogg, R. M.; Vlieg, E. Fast attrition-enhanced deracemization of naproxen by a gradual in situ feed. Angew. Chem. Int. Ed. 2009, 48, 4581-4583.

(27) Noorduin, W. L.; Vlieg, E.; Kellogg, R. M.; Kaptein, B. From Ostwald ripening to single chirality. Angew. Chem. Int. Ed. 2009, 48, 9600-9606.

(28) Viedma, C.; Michael, M. J.; Kahr, B.; Cintas, P. Enantiomer-Specific Oriented Attachment: Formation of Macroscopic Homochiral Crystal Aggregates from a Racemic System. Angew. Chem. Int. Ed. 2013, 52, 10545-10548.

(29) Su, Q.; Nagy, Z. K.; Rielly, C. D. Pharmaceutical crystallisation processes from batch to continuous operation using MSMPR stages: Modelling, design, and control. Chem. Eng. Process. 2015, 89, 41-53. 
(30) Mullin, J. W. Crystallization, 4th ed.; Butterworth-Heinemann: Oxford, UK, 2001.

(31) Yang, Y.; Song, L.; Gao, T.; Nagy, Z. K. Integrated Upstream and Downstream Application of Wet Milling with Continuous Mixed Suspension Mixed Product Removal Crystallization. Cryst. Growth Des. 2015, 15, 5879-5885.

(32) Acevedo, D.; Pena, R.; Barton, A.; Firth, P.; Nagy, Z. K. Evaluation of mixed suspension mixed product removal crystallization processes coupled with a continuous filtration system. Chem. Eng. 2016, 108, 212-219.

(33) Uwaha, M. A model for complete chiral crystallization. J. Phys. Soc. Jpn. 2004, 73, $2601-2603$.

(34) Saito, Y.; Hyuga, H. Chirality selection in crystallization. J. Phys. Soc. Jpn. 2005, 74, $535-537$.

(35) Saito, Y.; Hyuga, H. Chiral crystal growth under grinding. J. Phys. Soc. Jpn. 2008, rry, 113001.

(36) Noorduin, W. L.; van Enckevort, W. J. P.; Meekes, H.; Kaptein, B.; Kellogg, R. M.; Tully, J. C.; McBride, J. M.; Vlieg, E. The Driving Mechanism Behind AttritionEnhanced Deracemization. Angew. Chem. Int. Ed. 2010, 122, 8613-8616.

(37) Iggland, M.; Mazzotti, M. A population balance model for chiral resolution via Viedma ripening. Cryst. Growth Des. 2011, 11, 4611-4622.

(38) Gherase, D.; Conroy, D.; Matar, O. K.; Blackmond, D. G. Experimental and Theoretical Study of the Emergence of Single Chirality in Attrition-Enhanced Deracemization. Cryst. Growth Des. 2014, 14, 928-937.

(39) Iggland, M.; Müller, R.; Mazzotti, M. On the Effect of Initial Conditions in Viedma Ripening. Cryst. Growth Des. 2014, 14, 2488-2493. 
(40) Rougeot, C.; Hein, J. E. Application of Continuous Preferential Crystallization to Efficiently Access Enantiopure Chemicals. Org. Process Res. Dev. 2015,

(41) Steendam, R. R. E.; Dickhout, J.; van Enckevort, W. J. P.; Meekes, H.; Raap, J.; Rutjes, F. P. J. T.; Vlieg, E. Linear Deracemization Kinetics during Viedma Ripening: Autocatalysis Overruled by Chiral Additives. Cryst. Growth Des. 2015, 15, 1975-1982.

(42) Xiouras, C.; Van Aeken, J.; Panis, J.; Ter Horst, J. H.; Van Gerven, T.; Stefanidis, G. D. Attrition-enhanced deracemization of NaClO3: Comparison between ultrasonic and abrasive grinding. Cryst. Growth Des. 2015, 15, 5476-5484.

(43) Ratke, L.; Voorhees, P. W. Growth and coarsening: Ostwald ripening in material processing; Springer: New York City, NY, 2013.

(44) Ostwald, W. Studien über die Bildung und Umwandlung fester Körper. Z. Phys. Chem. 1897, 22, 289-330.

(45) Vetter, T.; Iggland, M.; Ochsenbein, D.; Hänseler, F.; Mazzotti, M. Modeling Nucleation, Growth, and Ostwald Ripening in Crystallization Processes: A Comparison between Population Balance and Kinetic Rate Equation. Cryst. Growth Des. 2013, 13, 4890-4905.

(46) Kashchiev, D.; Van Rosmalen, G. M. Review: nucleation in solutions revisited. Cryst. Res. Technol. 2003, 38, 555-574.

(47) Iggland, M.; Mazzotti, M. Population Balance Modeling with Size-Dependent Solubility: Ostwald Ripening. Cryst. Growth Des. 2012, 12, 1489-1500.

(48) Ochsenbein, D. R.; Vetter, T.; Schorsch, S.; Morari, M.; Mazzotti, M. Agglomeration of Needle-like Crystals in Suspension: I. Measurements. Cryst. Growth Des. 2015, 15, $1923-1933$. 
(49) Ochsenbein, D. R.; Vetter, T.; Morari, M.; Mazzotti, M. Agglomeration of Needle-like Crystals in Suspension: II. Modeling. Cryst. Growth Des. 2015, 15, 4296-4310.

(50) Lindenberg, C.; Schöll, J.; Vicum, L.; Mazzotti, M.; Brozio, J. L-glutamic acid precipitation: agglomeration effects. Cryst. Growth Des. 2008, 8, 224-237.

(51) Hounslow, M. J. A discretized population balance for continuous systems at steady state. AIChE J. 1990, 36, 106-116.

(52) Jacques, J.; Collet, A.; Wilen, S. H. Handbook of Industrial Crystallization, 2nd ed.; Butterworth-Heinemann: Oxford, UK, 2002.

(53) Agrawal, S.; Paterson, A. Secondary Nucleation: Mechanisms and Models. Chem. Eng. Commun. 2015, 202, 698-706.

(54) Ramkrishna, D. Population Balances: Theory and Applications to Particulate Systems in Engineering; Academic Press: Cambridge, MA, 2000.

(55) Tsogoeva, S.; Wei, S.; Freund, M.; Mauksch, M. Generation of Highly Enantioenriched Crystalline Products in Reversible Asymmetric Reactions with Racemic or Achiral Catalysts. Angew. Chem. Int. Ed. 2009, 48, 590-594.

(56) Li, W.; Spix, L.; de Reus, S.; Meekes, H.; Kramer, H.; Vlieg, E.; ter Horst, J. Deracemization of a Racemic Compound via Its Conglomerate-Forming Salt Using Temperature Cycling. Cryst. Growth Des. 2016, 16, 5563-5570.

(57) Suwannasang, K.; Flood, A. E.; Rougeot, C.; Coquerel, G. Using programmed heatingcooling cycles with racemization in solution for complete symmetry breaking of a conglomerate forming system. Cryst. Growth Des. 2013, 13, 3498-3504.

(58) Suwannasang, K.; Flood, A. E.; Coquerel, G. A novel design approach to scale up the temperature cycle enhanced deracemization process: coupled mixed-suspension vessels. Cryst. Growth Des. 2016, 16, 6461-6467. 


\section{For Table of Contents Use Only}

Title: Model-based analysis of continuous crystallization/reaction processes separating conglomerate forming enantiomers

Authors: Till Köllges, Thomas Vetter

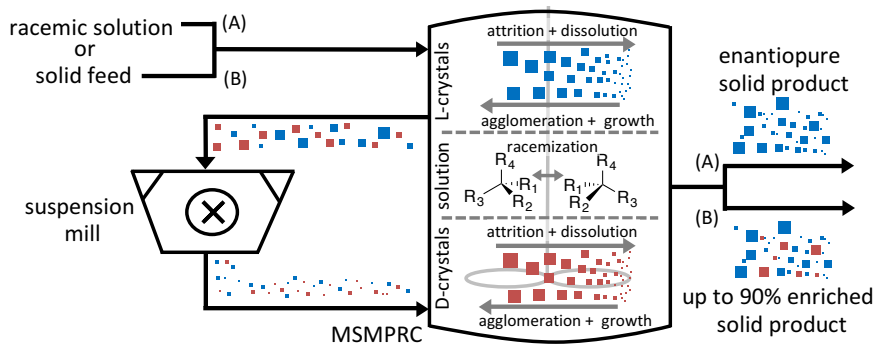

Synopsis: Two continuous processes combining crystallization, milling and racemization in solution are presented and applied to the separation of conglomerate forming enantiomers. The processes are fed either with a racemic solution or a racemic mixture of crystals. We show that the solution-fed process reaches full enantiopurity and high yields, while the solidfed process is limited to roughly $90 \%$ enantiopurity. 